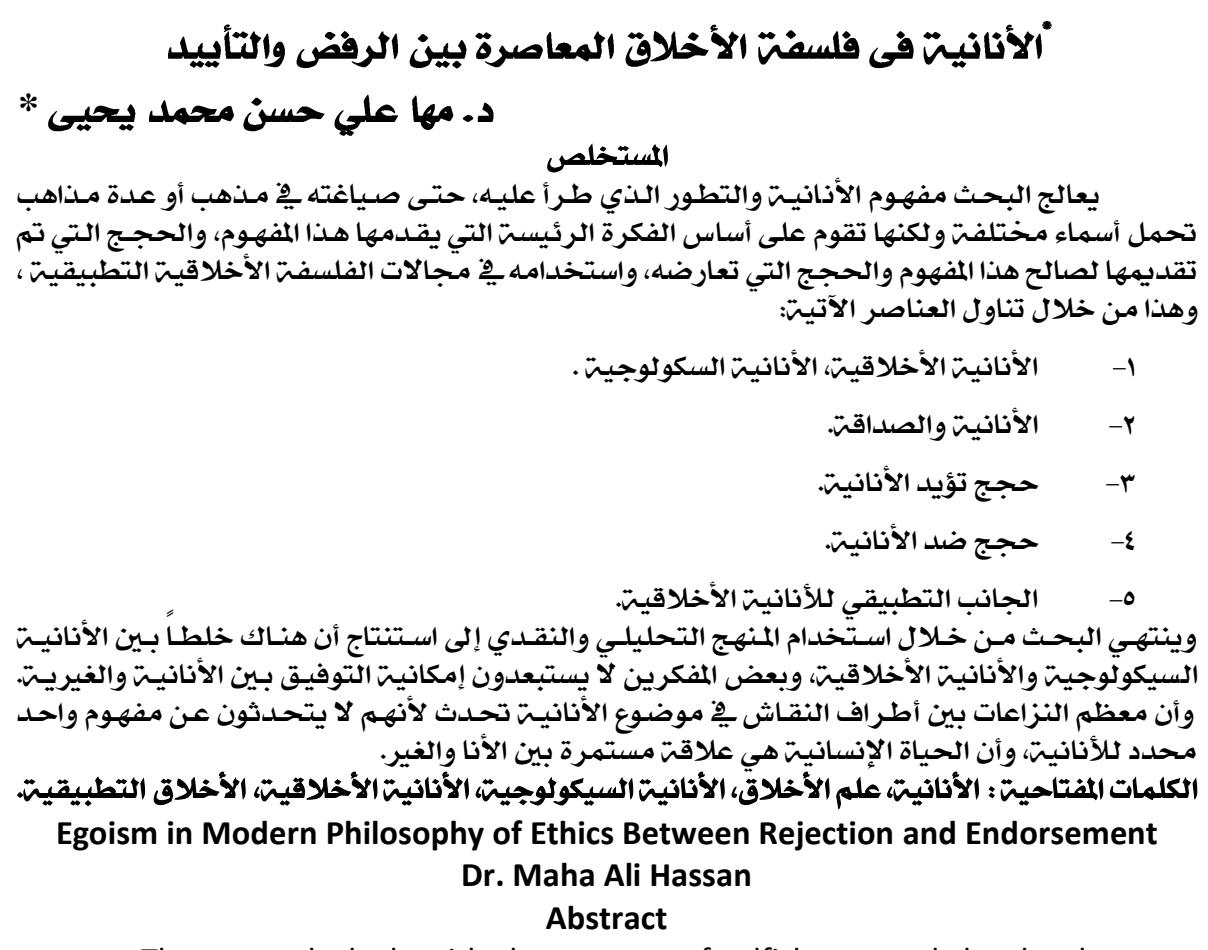

The research deals with the concept of selfishness and the development that occurred to it, until it was formulated in one or several doctrines bearing different names but based on the main idea presented by this concept, the arguments that were presented in favor of this concept and the arguments that

• إنانيتّ: أثرة: حب النفس، ويطلق أخلاقيًا على من لا يهدف إلا لنفعه الخاص، ويقابل الإيثار.

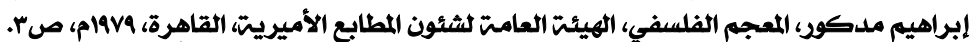

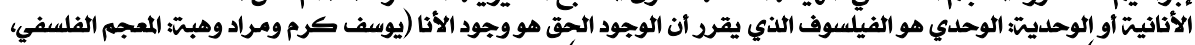

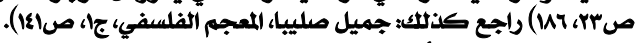

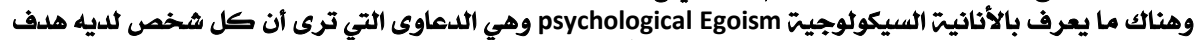

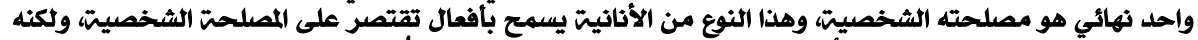

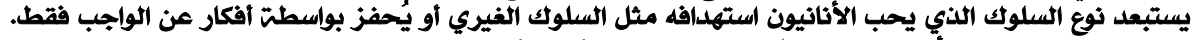

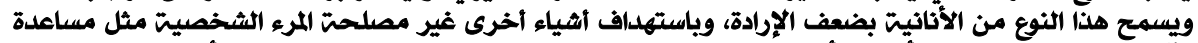

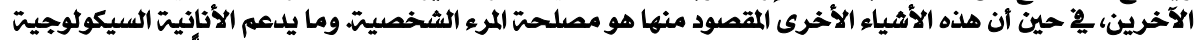

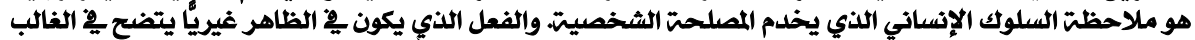

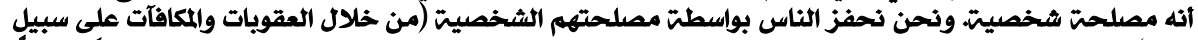

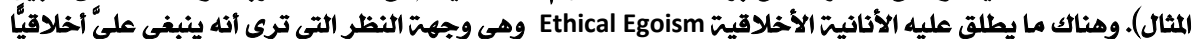

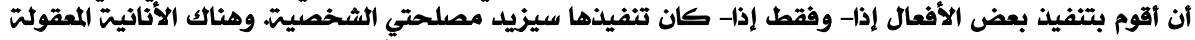
Rational Egoism

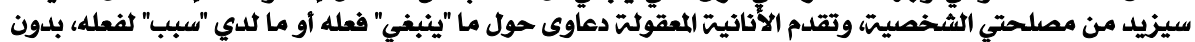
حصر "ينبغي" أو "سبب" ملى الناحيت الأخلاقيتة وتصنيت https://plato.stanford.edu/entries/egoism/(2/10/2019) • ملدربس فلسفت الأخلاق بكليت الآداب -جامعت الموادي الجلديل 10 
oppose it, and its use in the fields of applied moral philosophy, and discussing the following points:

1- Ethical egoism, psychological egoism.

2- Egoism and friendship.

3- Arguments in favor Egoism.

4- Arguments against Egoism.

5- The practical side of moral Egoism.

The research ends through the use of the analytical and critical Method to the conclusion that there is a confusion between psychological egoism and moral egoism, and some thinkers do not rule out the possibility of reconciling between Egoism and Altruism. And that most of the disputes are between the parties to the discussion on the subject of egoism it occurs because they do not talk about a single specific concept of egoism, and that human life is an ongoing relationship between the ego and others.

Keywords: Selfishness-Ethics-psychological egoism -moral Egoism- Applied ethics.

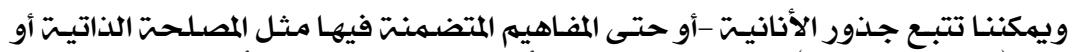

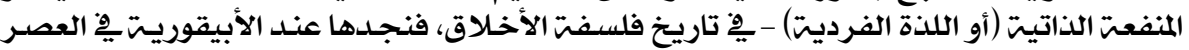

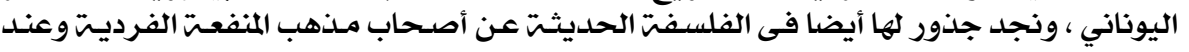

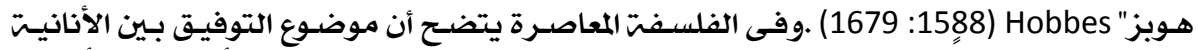

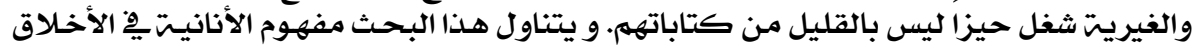

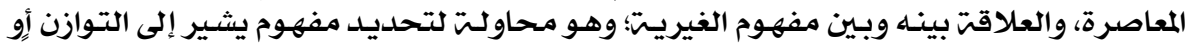

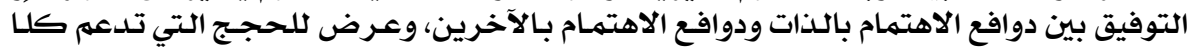

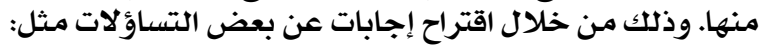
ا- ما مدى إمكانيت التوفيق بين الأنانيت والغيريتى

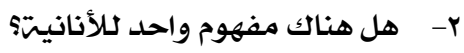

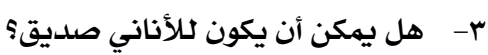

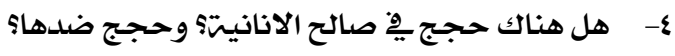
هـ هل هنالك إمكانيت لتطبيق مفهوم الانانيت ِِْ مجال الأخلاق التطبيقيت؟ الأنانيت والفيريت

يعترض فلاسفت الأخلاق المعاصرون على مصطلح "الغيريت" Altruism أو بشكل أشثر

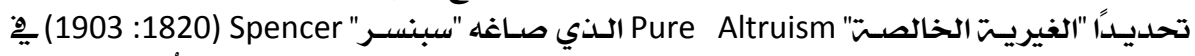

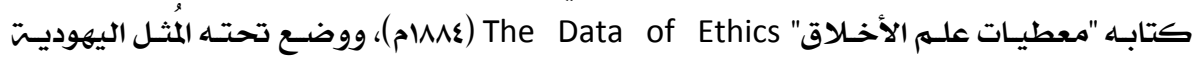

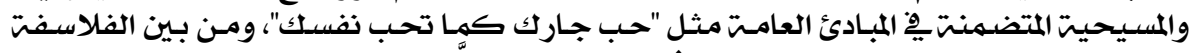

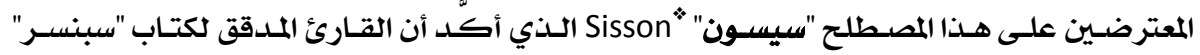




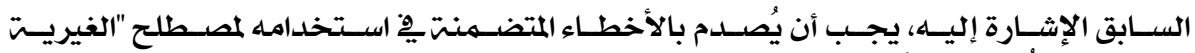

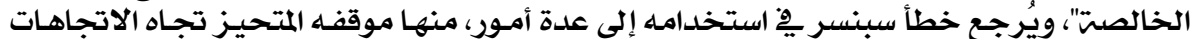

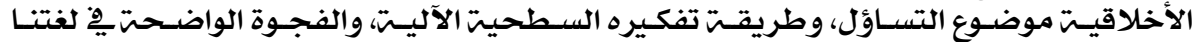

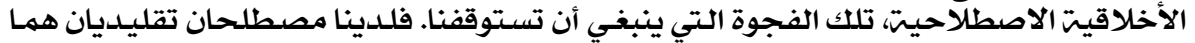

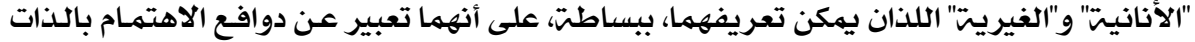

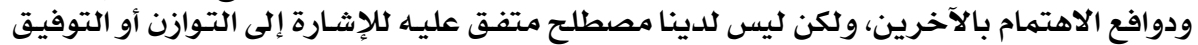

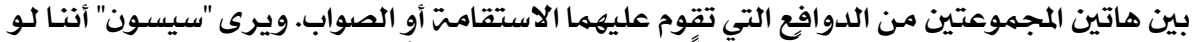

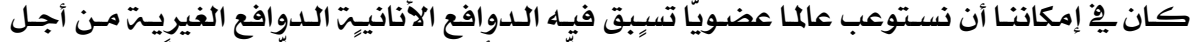

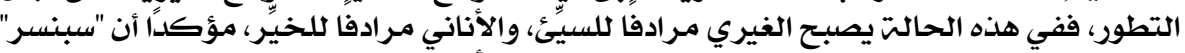

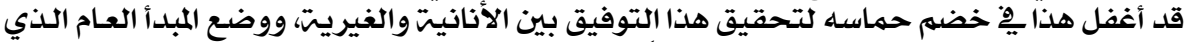

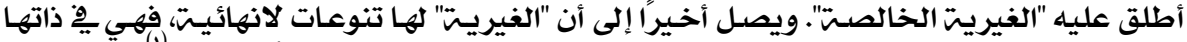

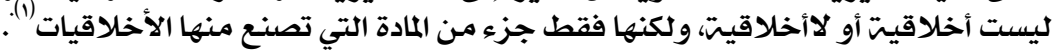

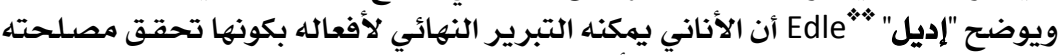

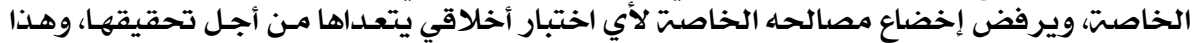

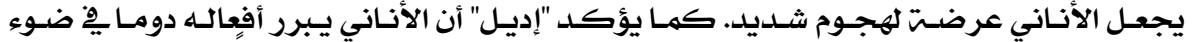

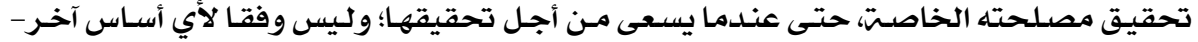

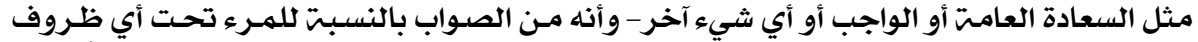

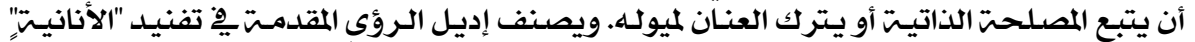

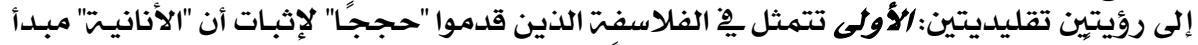

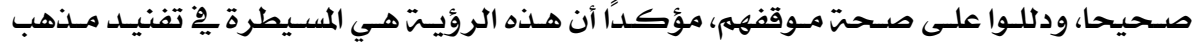

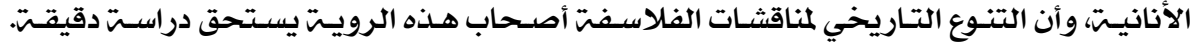

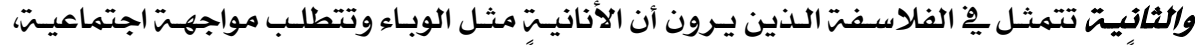

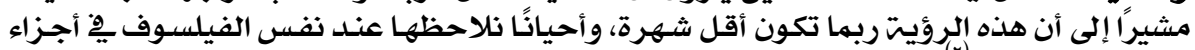

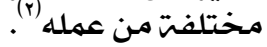

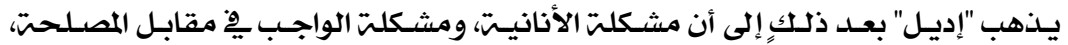

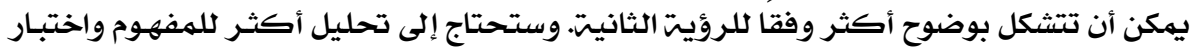

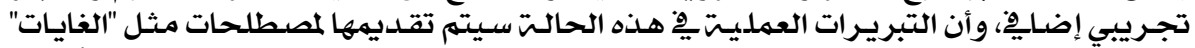

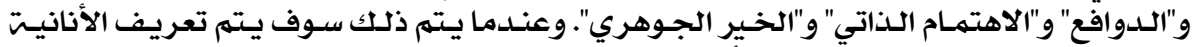

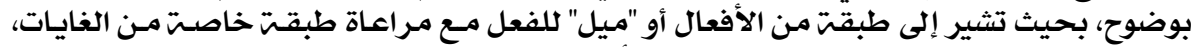

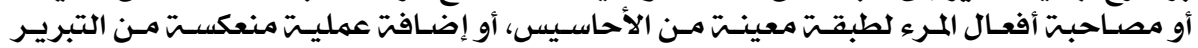

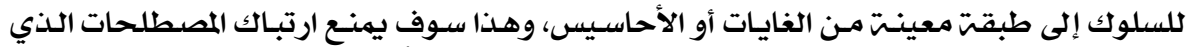

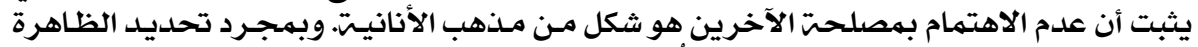

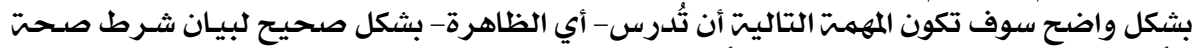

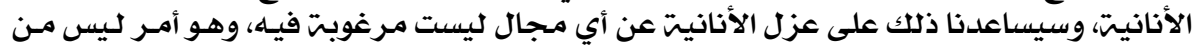

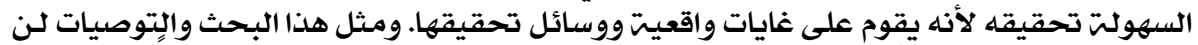

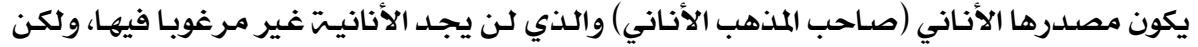

https://www.umt.edu/president/people/pastpresidents/sisson.php (2/10/2019)

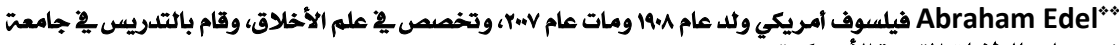
نيويورك بالولايات المتحدة الأمريكيت. https://www.oxfordreference.com/view/10.1093/oi/authority.20110915125649353 http://web.gc.cuny.edu/Philosophy/edel.html (2/10/2019) 


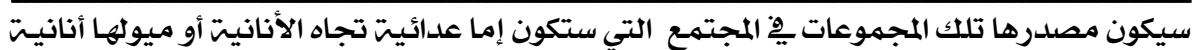

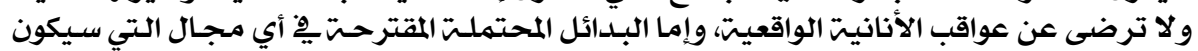

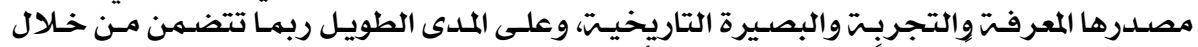

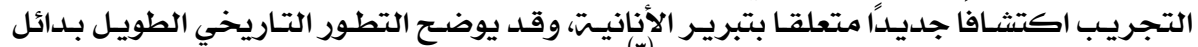

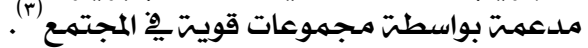

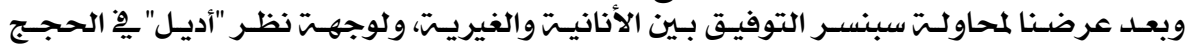

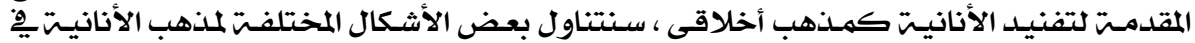

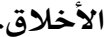

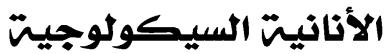

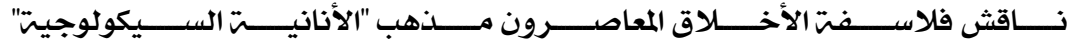

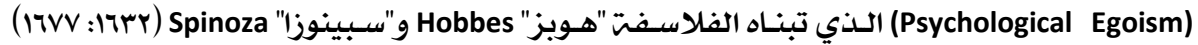

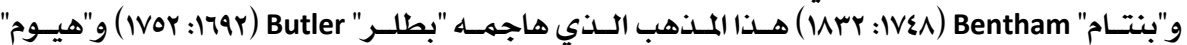

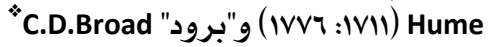

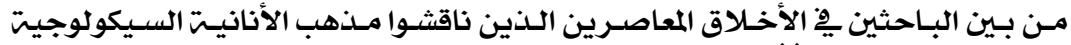

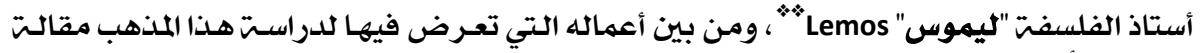

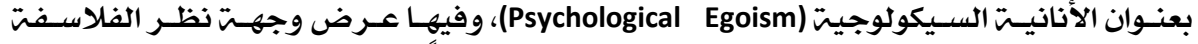

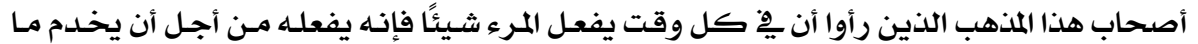

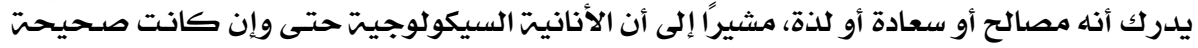

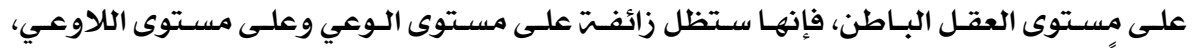

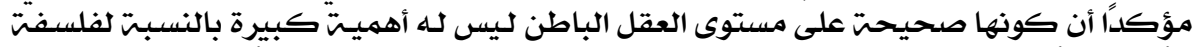

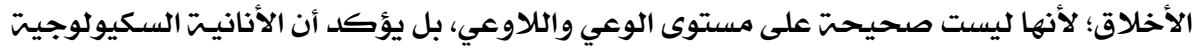

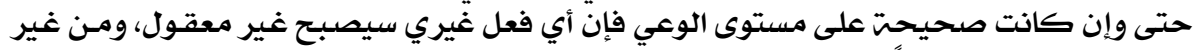

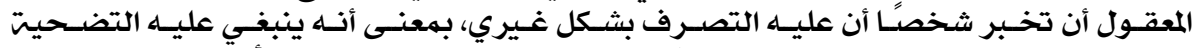

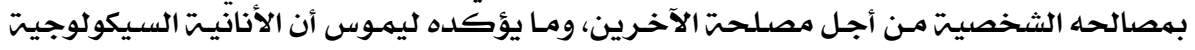

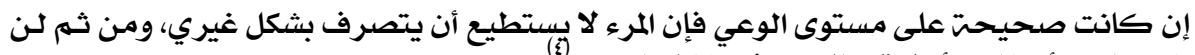

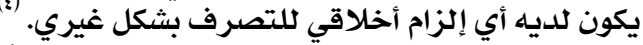

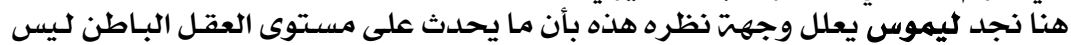

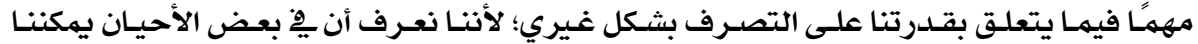

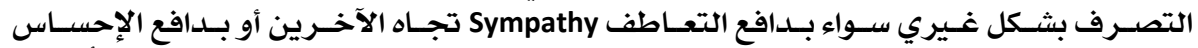

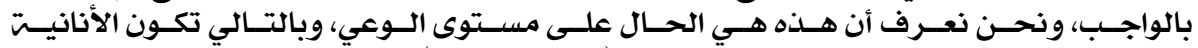

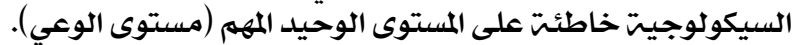

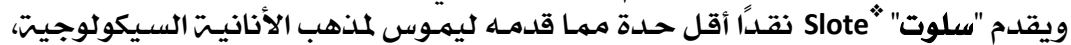

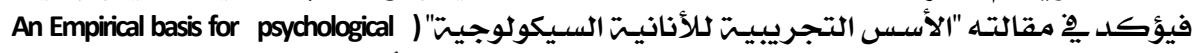

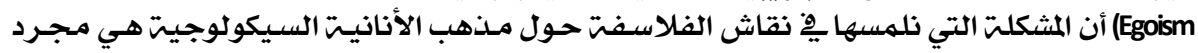

وال1887-1971) C.D.Broad * Russell, Locke Paul Edwards,The Encyclopedia of philosophy,Vol.1, Macmillian, London,1967, p396, 399. أستاذ الفلسفت بجامعت ميامي بالولايات المتحدة الأمريكيت. Lemos .M Ramon ** https://www.amazon.com \& Neomedieval-Essay-Philosoph... (3/10/2019) Michael Slote • http://www.phil.arts.cuhk.edu.hk/web/tcivp/michael-slote/(25/10/2019) 


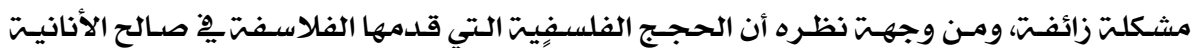

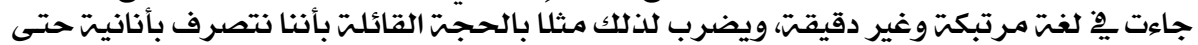

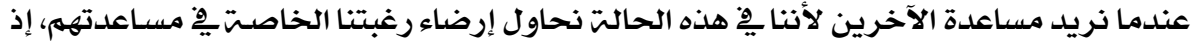

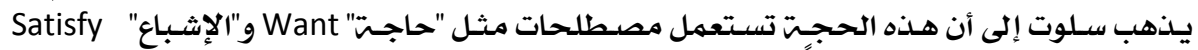

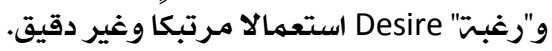

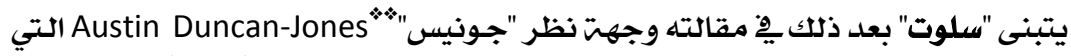

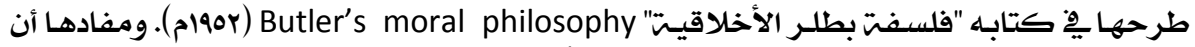

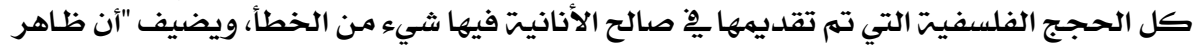

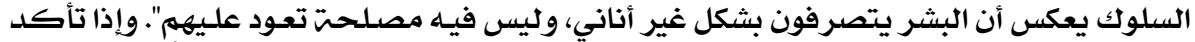

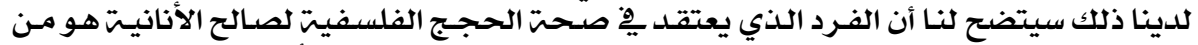

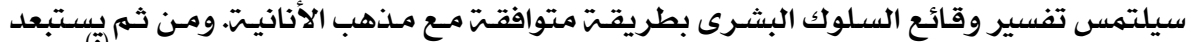

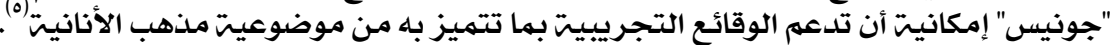

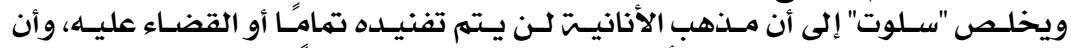

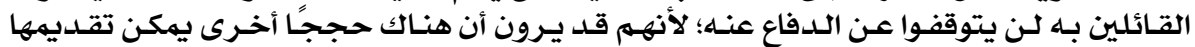

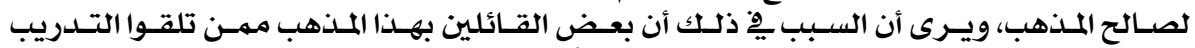

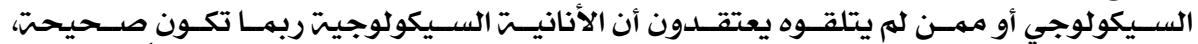

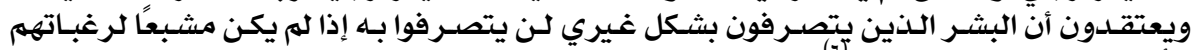

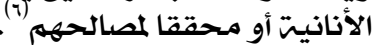
ومن الأشكال الأخرى التي يتضمنها مذهب الأنانيت مـذهب الأنانيت الأخلاقيت.

الأنانيت الأخلاقيت

تعرَّ فلاسفت الأخلاق المعاصـرون لنظريـت "الأنانيـت الأخلاقيـت" (Ethical Egoism) ف"براندت"

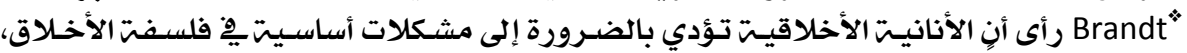

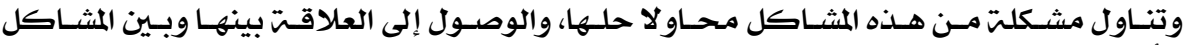

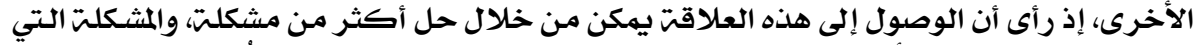

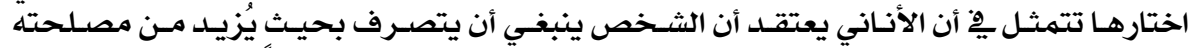

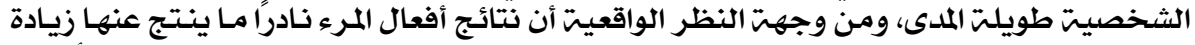

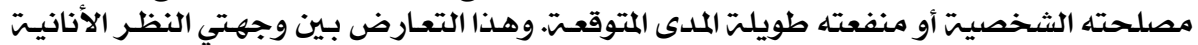

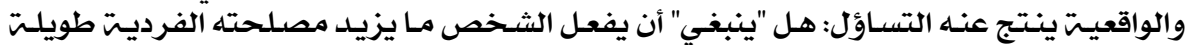

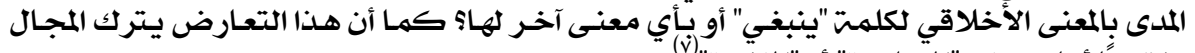

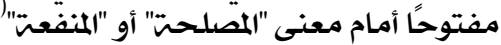

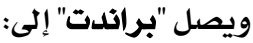

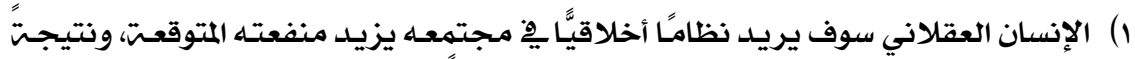

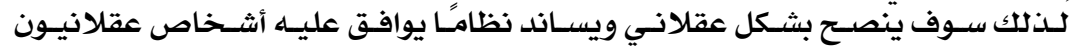

Austin Duncan-Jones * هو فيلسوف بريطاني ولد عام 1941م ومات عام 197Vم. https://www.ukwhoswho.com/search?q=Austin+DuncanJones\&searchBtn=Search\&isQuickSearch=true (25/10/2019)

Brandt .B Richard • https://www.cambridge.org/core/services/aop-cambridgecore/content/view/S0953820800006300 (25/10/2019) 


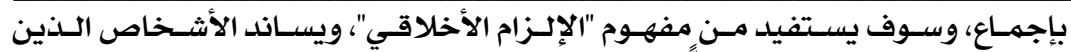

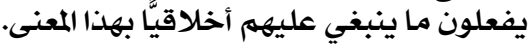

r) هناك مصادر متنوعت من التحفيز لتأكيــ متطلبـات هـذا النظام حتى عنـدما يكون فعل هذا يتعارض مـع مصائح الآخرين.

r) والشخص يتصرف بشكل يزيد منفعته المتوقعت عندما يمنح دعمه للنظام الأخلاقي الذي إني

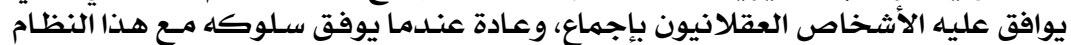

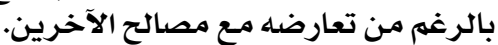

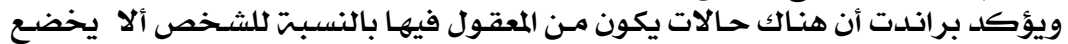

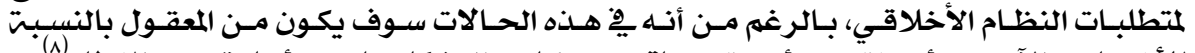

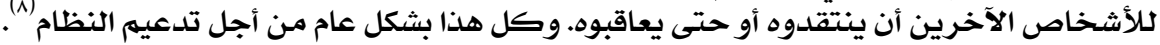

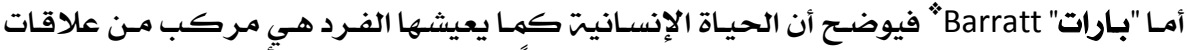

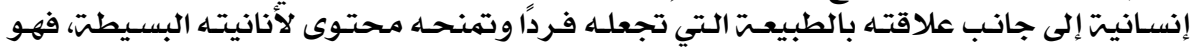

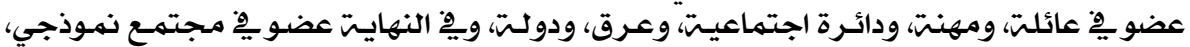

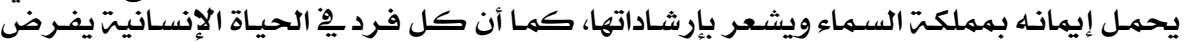

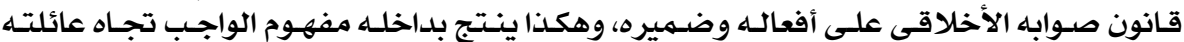
وأصدقائه وبلده وربه الانده

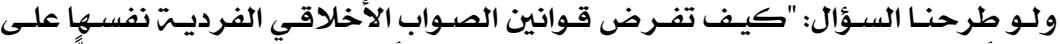

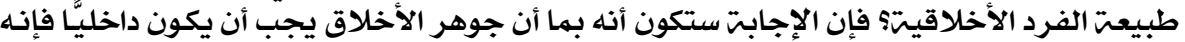

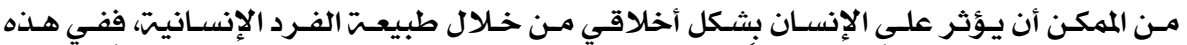

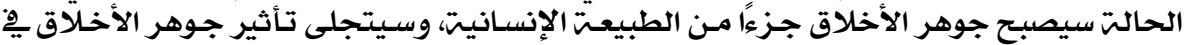

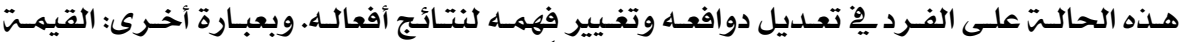

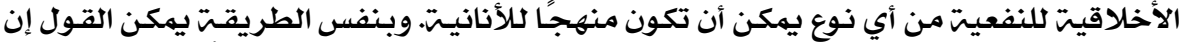

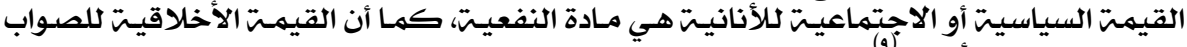

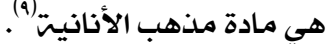

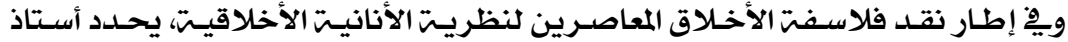

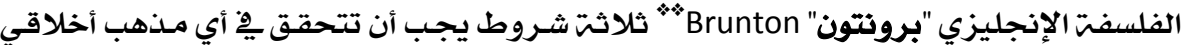

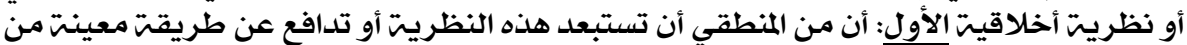

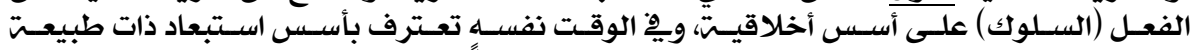

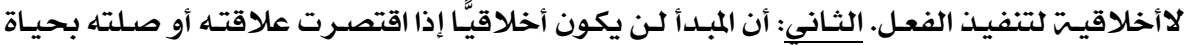

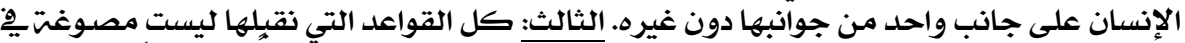

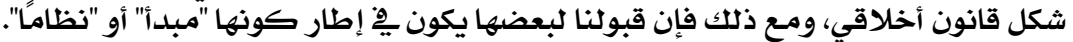

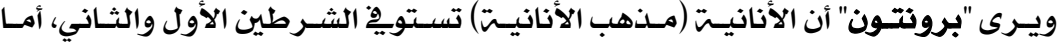

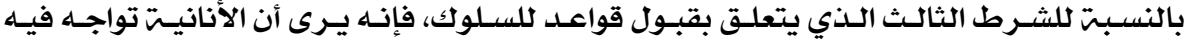

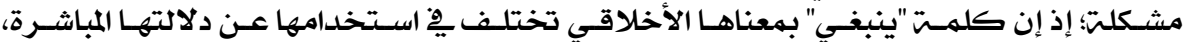

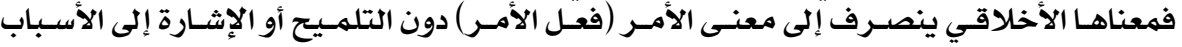

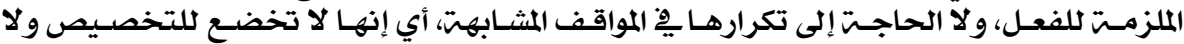

Alfred Barratt • https://oxfordindex.oup.com/oi/viewoverview/10.1093\$002foi\$002fauthority.201 $10803095448519(25 / 10 / 2019)$

هو أستاذ الفلسفت بجامعت جنوب ويلز يإنجلترا. 


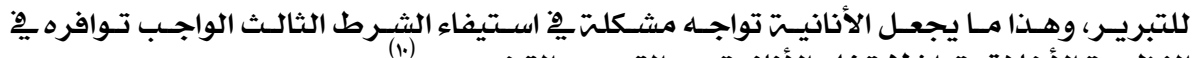

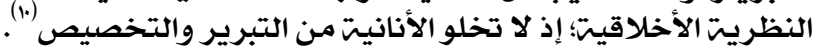

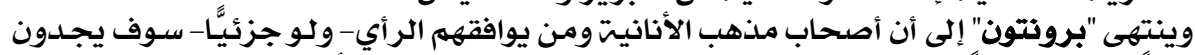

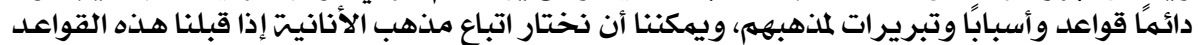

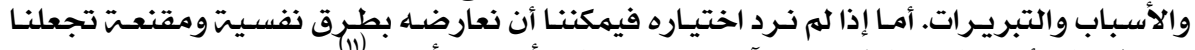

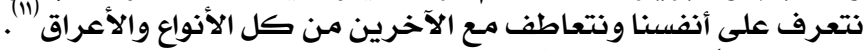

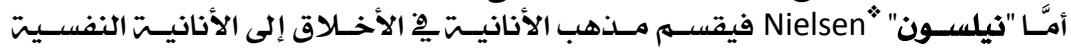

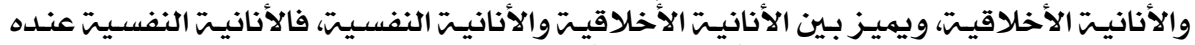

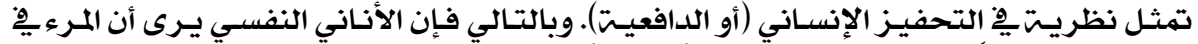

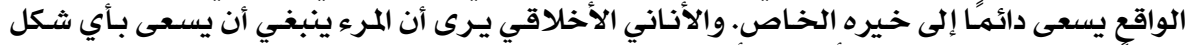

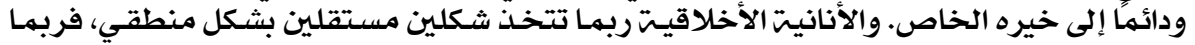

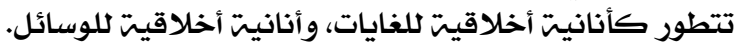

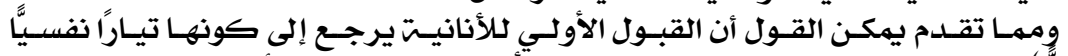

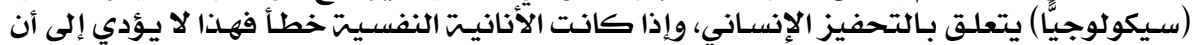

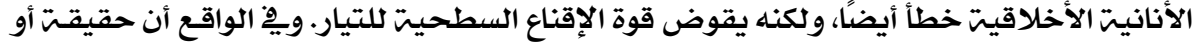

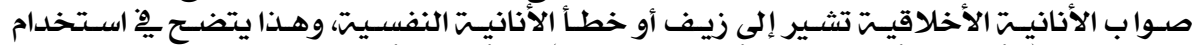

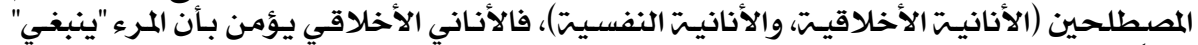

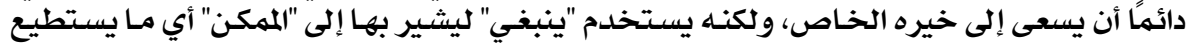

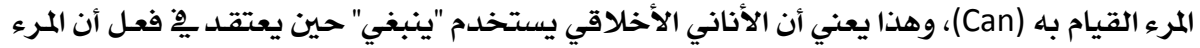

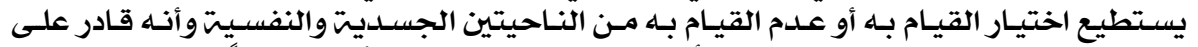

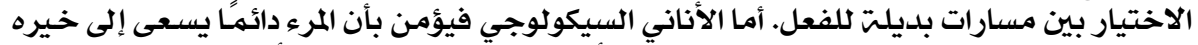

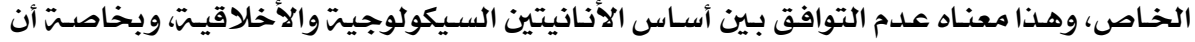

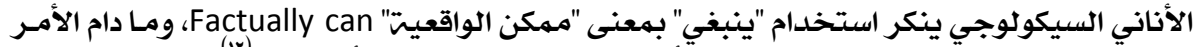

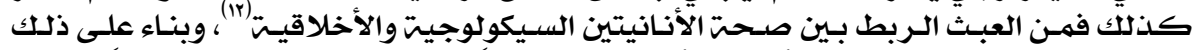

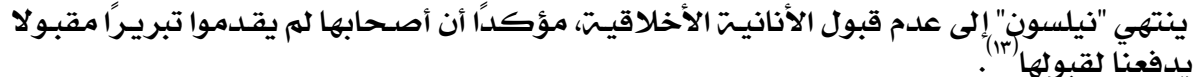

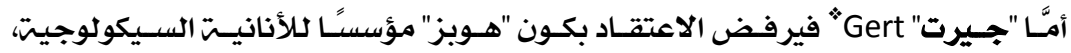

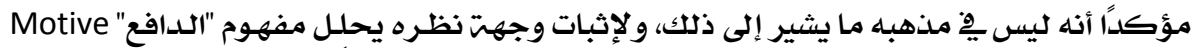

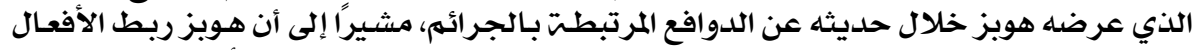

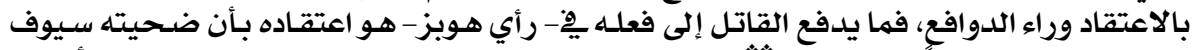

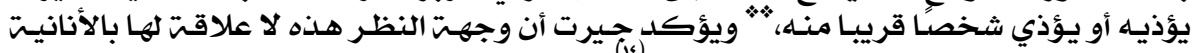

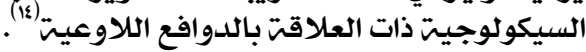

Kai Nielsen •

https://www.kainielsen.org 10/11/2019

https://phil.ucalgary.ca/profiles/kai-nielsen

Bernard Gert • http://www.dartmouth.edu/ gert/ 11/11/2019

http://www.chapelhillnews.com/2012/01/01/69116/bernard-gert.html

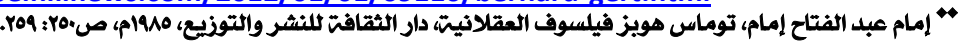




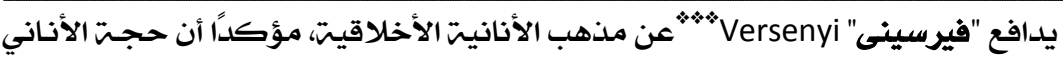

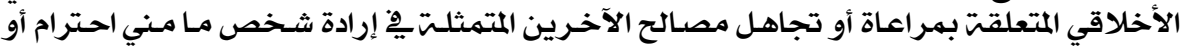

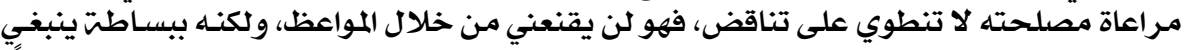

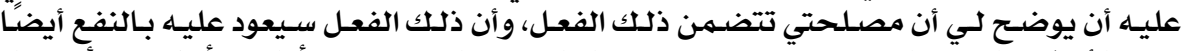

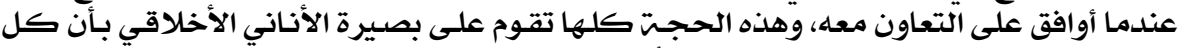

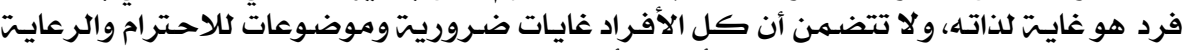

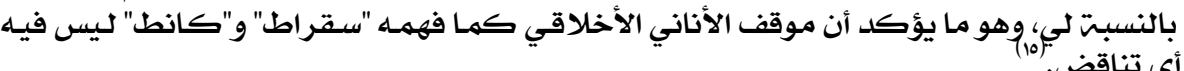

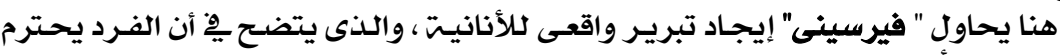

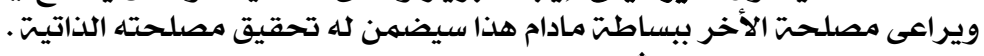

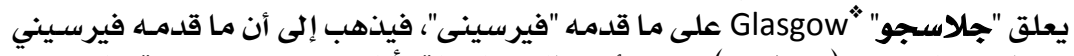

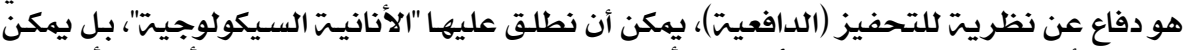

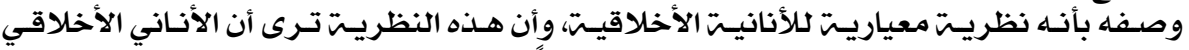

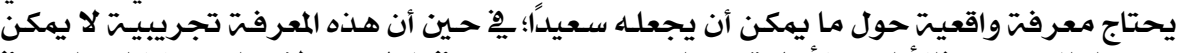

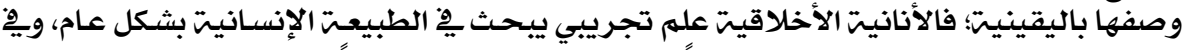

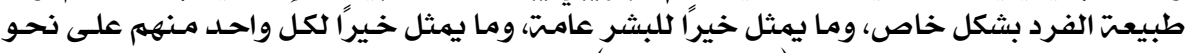

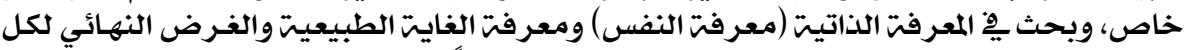

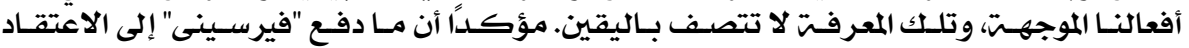

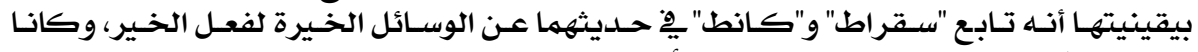

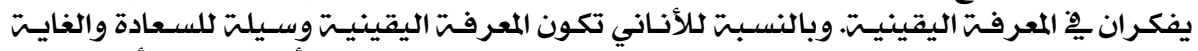

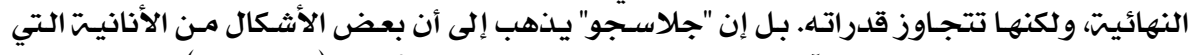

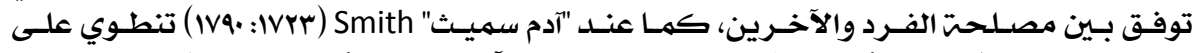

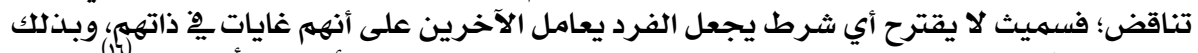

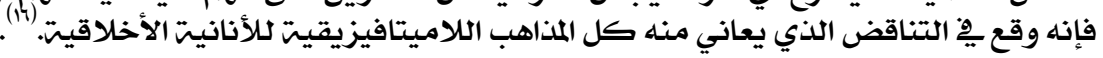

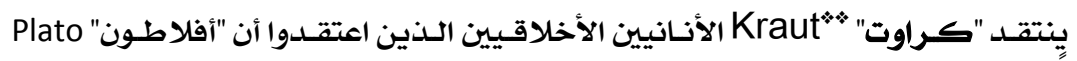

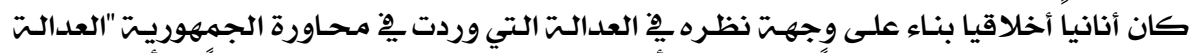

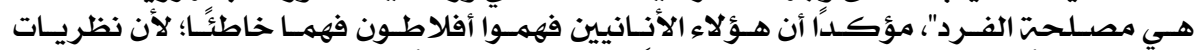

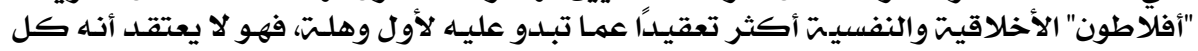

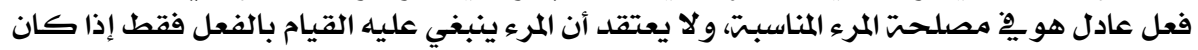

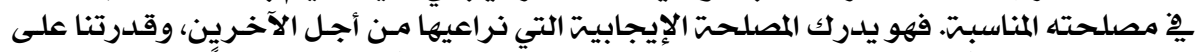

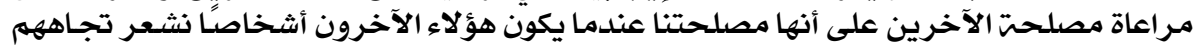

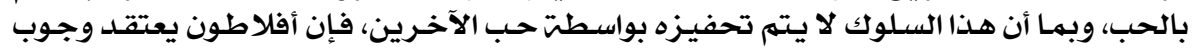

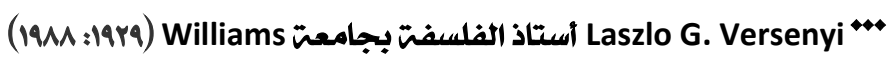
https://www.nytimes.com/1988/02/26/obituaries/laszlo-g-versenyi-philosophyprofessor-59.html 11/11/2019

W. D. Glasgow *

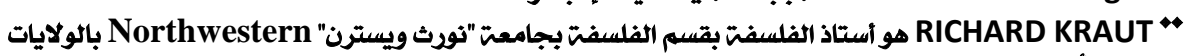
المتحدة الأمريكيتً.

https://philosophy.northwestern.edu/people/continuing-faculty/krautrichard.html 1/11/2020 


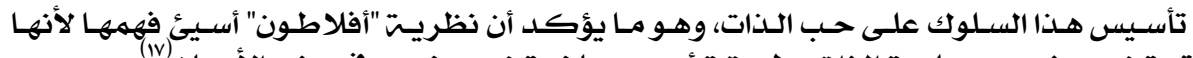

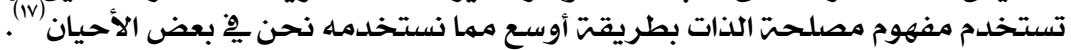

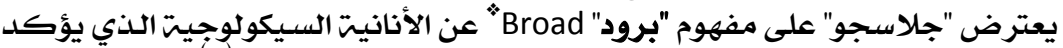

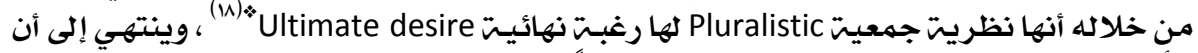

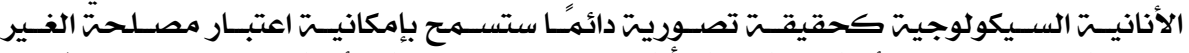

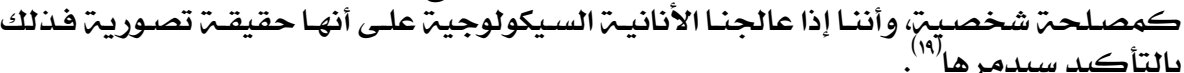

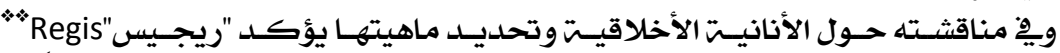

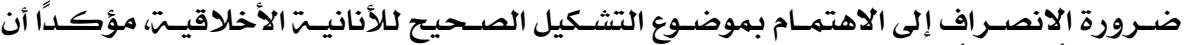

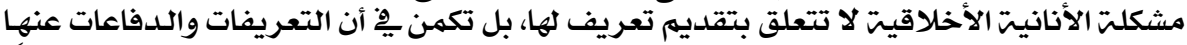

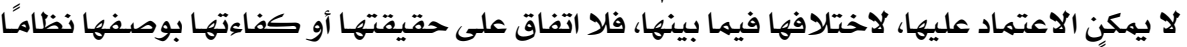

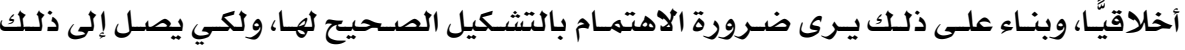

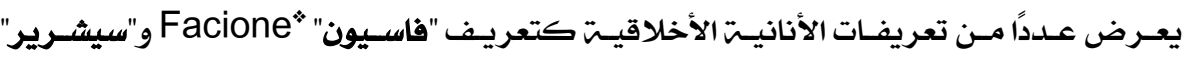

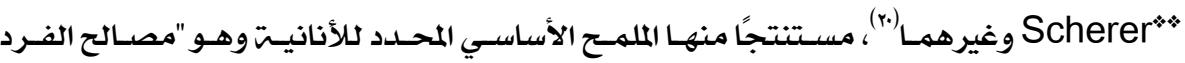

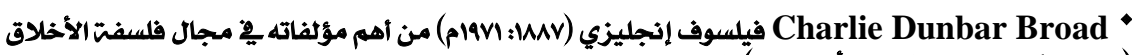
(خمست أنواع من النظريت الأخلاقيت) (عام. (خوفام.

https://plato.stanford.edu/entries/broad/ 14/11/2019

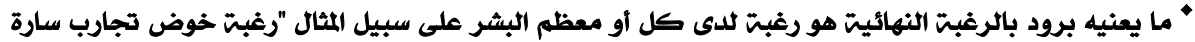

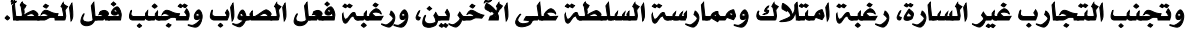

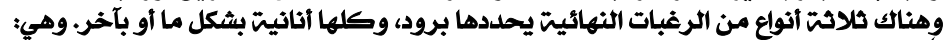

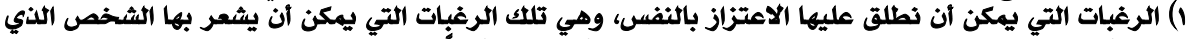

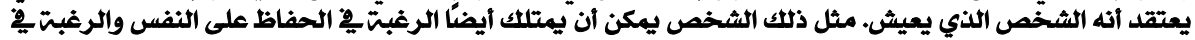

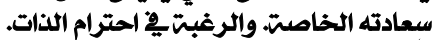

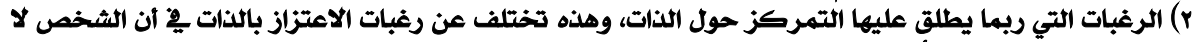

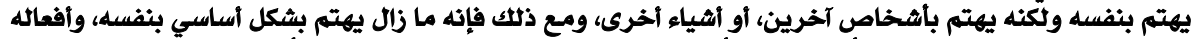

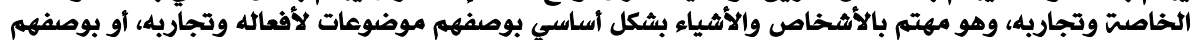

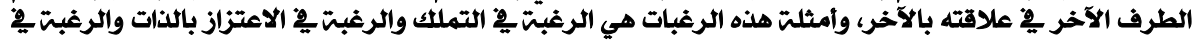

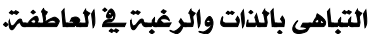

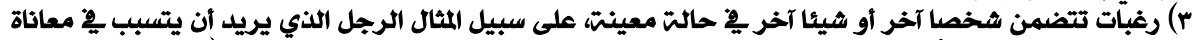

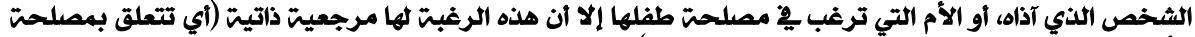

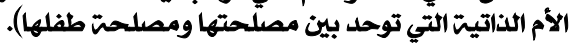

Broad C.D,"Egoism as A theory of Human Motives" in Ethics and the history of philosophy,London:Routledg\&Kegan paul,1952,p.220

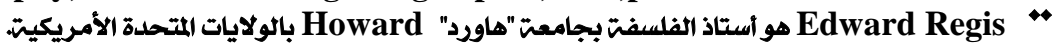
Regis, Edward. "Aristotle's 'Principle of Individuation ”.'Phronesis ،vol. 21, no. 2, 1976, pp. 157-166 JSTOR ،www.jstor.org/stable/4181987. 14/11/2019

Peter A. Facione * http://independent.academia.edu/PeterFacione/CurriculumVitae 14/11/2019

هو أستاذ الفلسفت بجامعت "بولينج جرين" Bowling Green بالولايات المتحدة 


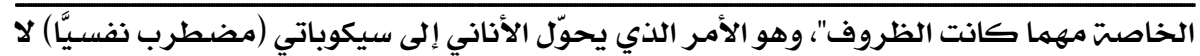

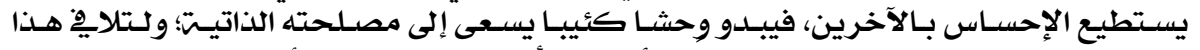

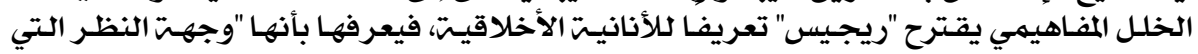

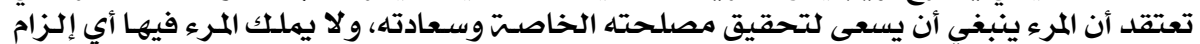

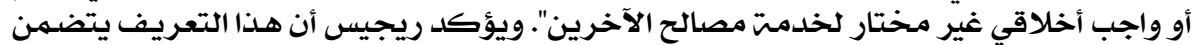

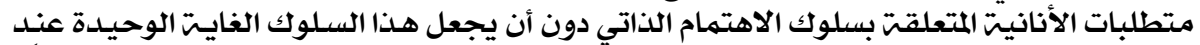

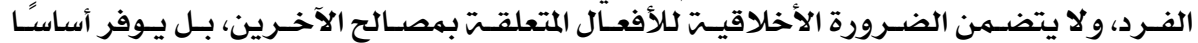

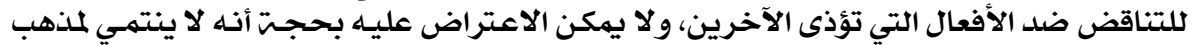

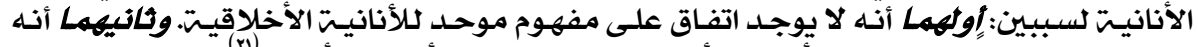

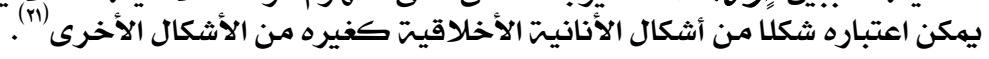

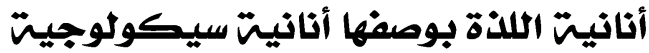

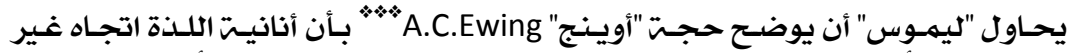

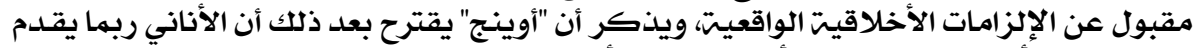

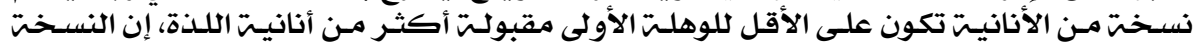

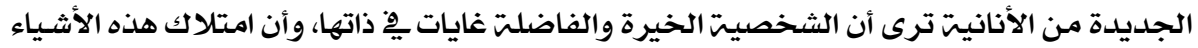

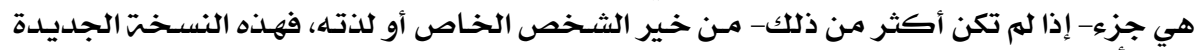

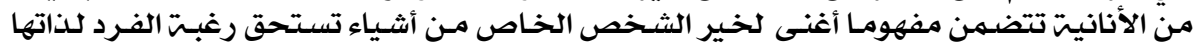

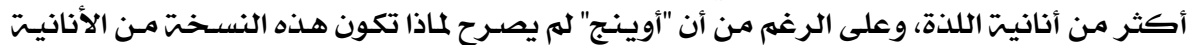

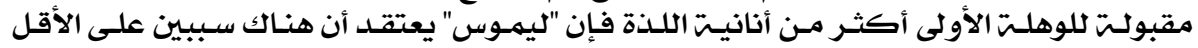

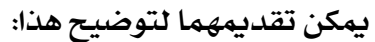

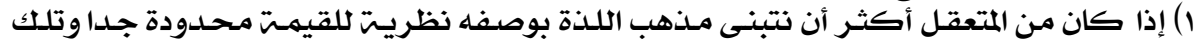

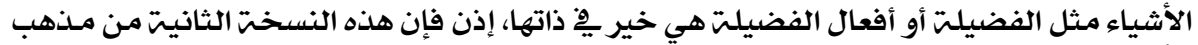

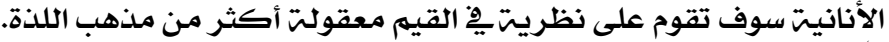

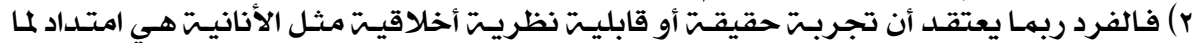

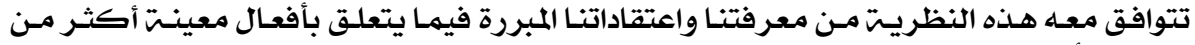

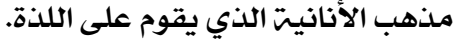

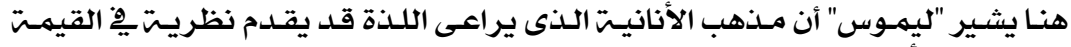

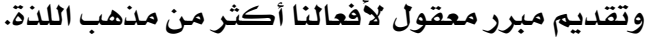

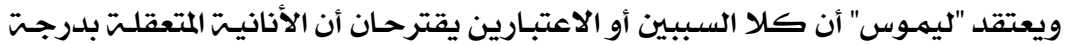

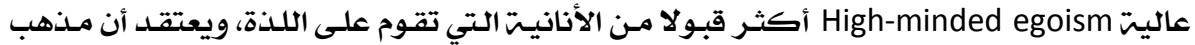

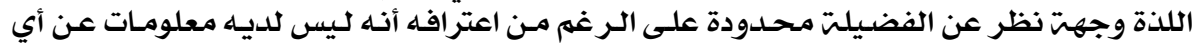

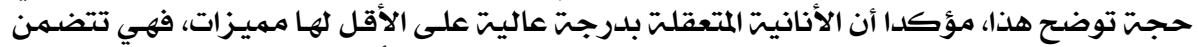

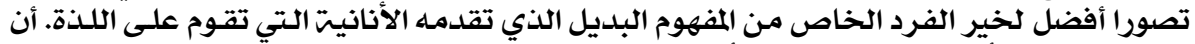

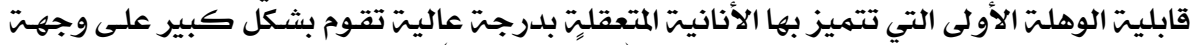

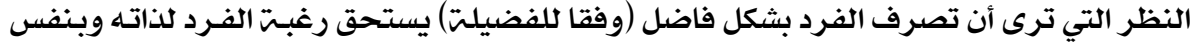

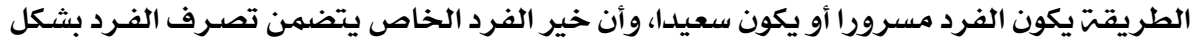

https://www.bgsu.edu/arts-and-sciences/philosophy/faculty-and-staff/dschere.html $14 / 11 / 2019$

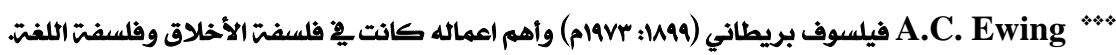
https://www.britannica.com/biography/A-C-Ewing 10/3/2020 


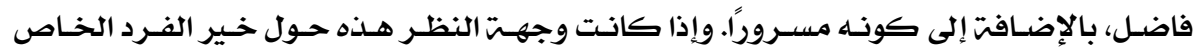

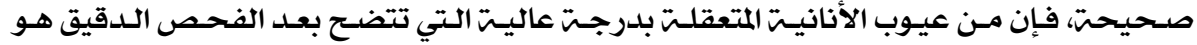

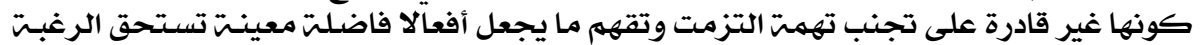

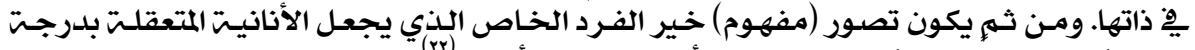

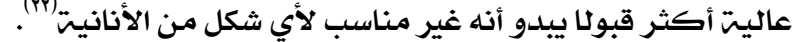

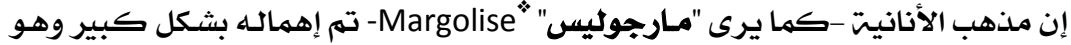

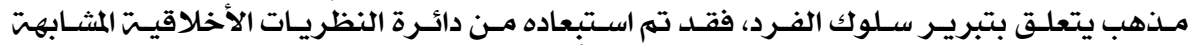

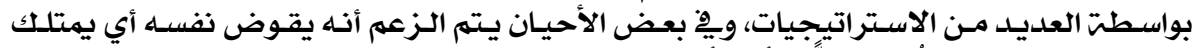

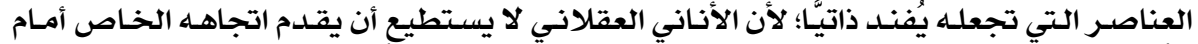

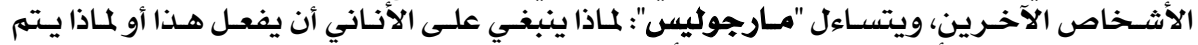

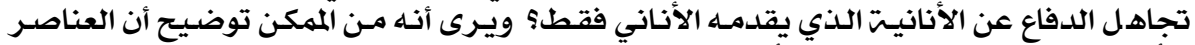

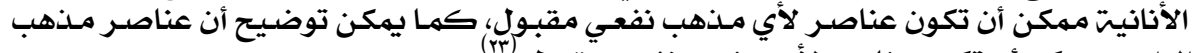

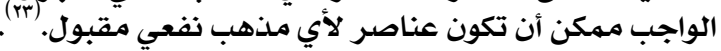

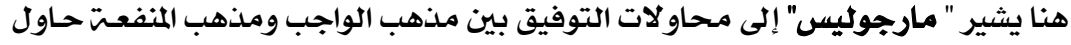

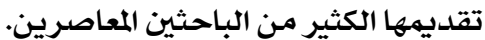

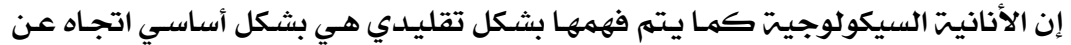

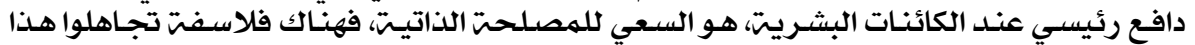

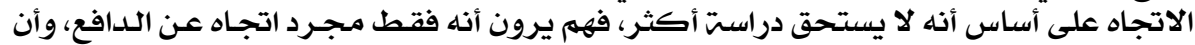

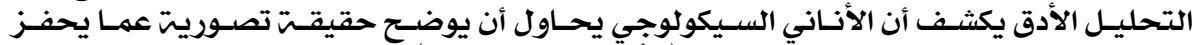

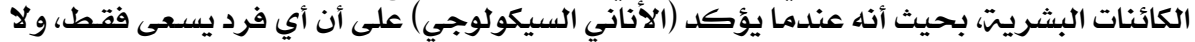

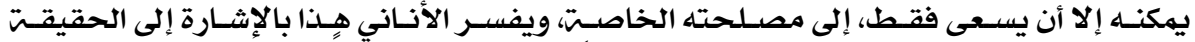

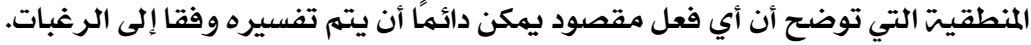

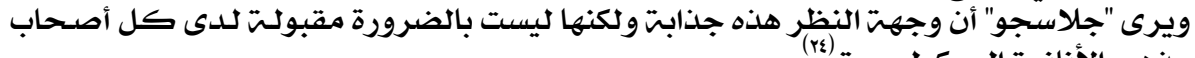

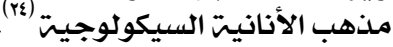

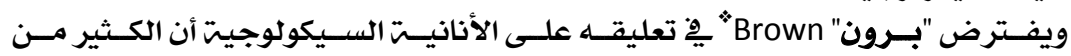

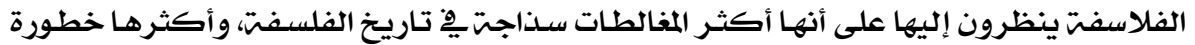

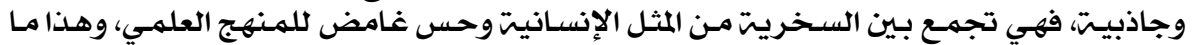

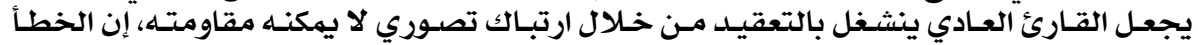

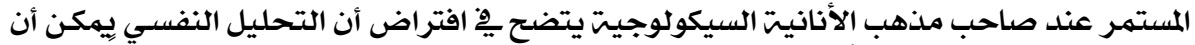

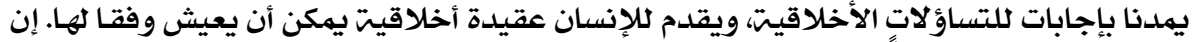

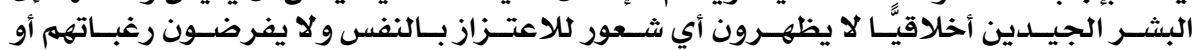

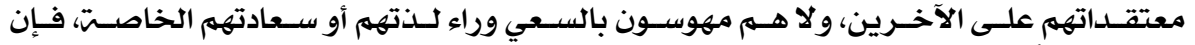

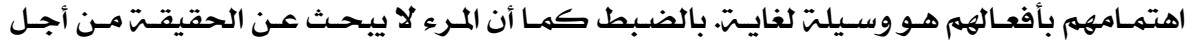

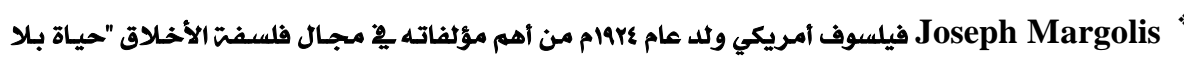

Life Without Principles: Reconciling Theory and Practice مبادئ: التصالح بين النظريت والممارستر" عام 1997ام. https://liberalarts.temple.edu/academics/faculty/margolis-joseph?action 15/11/2019

http://ecommons.udayton.edu/cgi/viewcontent.cgi?article=1004\&context=phl_f ac_pub 


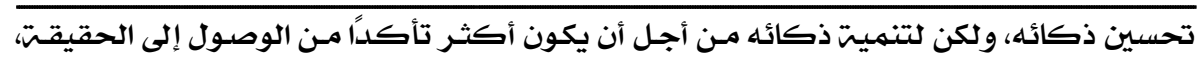

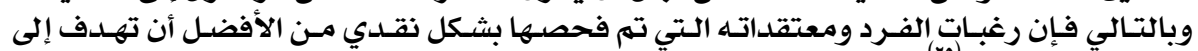

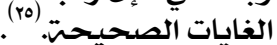

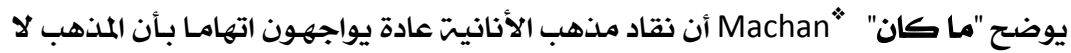

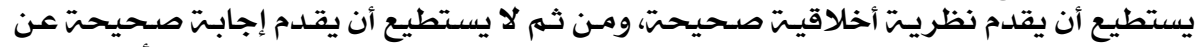

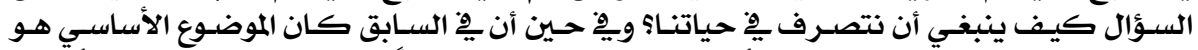

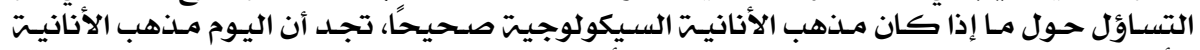

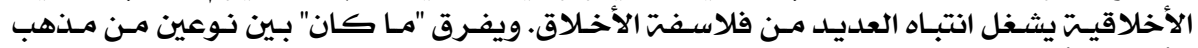

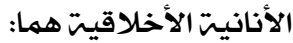
أ) مذهب الأنانيت الأخلاقيت الذانية الأني الذيى يتعلق بالفرد الواحد.

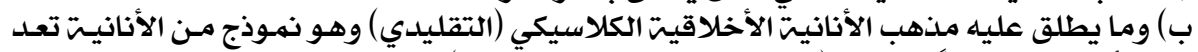

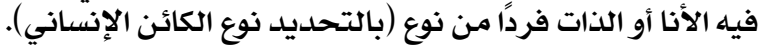

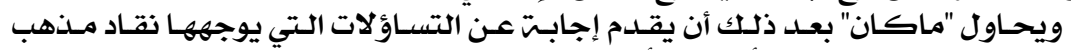

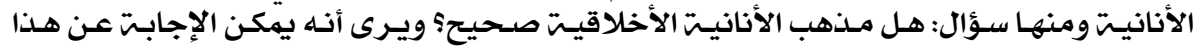

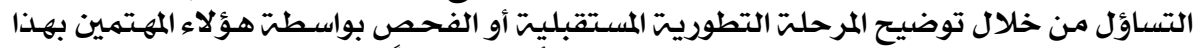

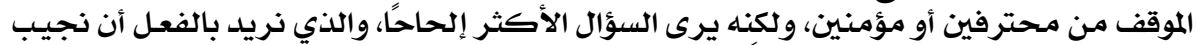

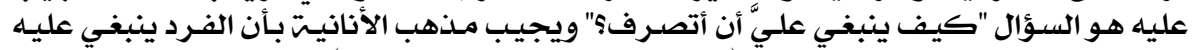

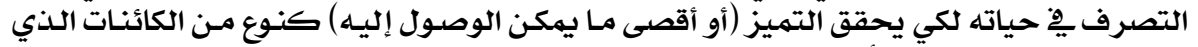

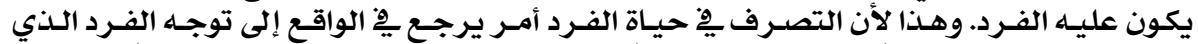

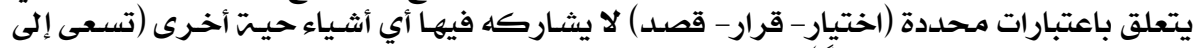

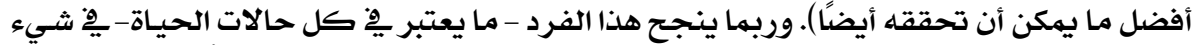

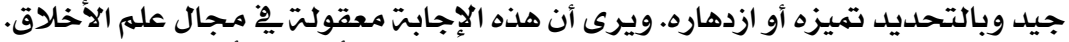

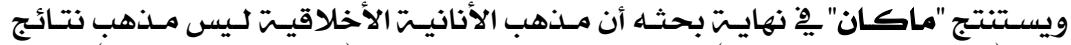

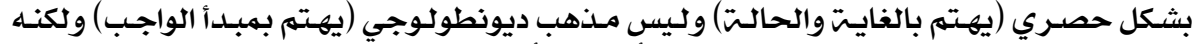

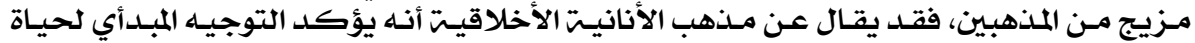

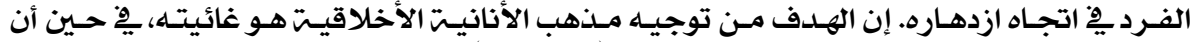

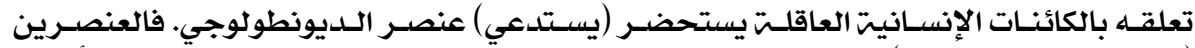

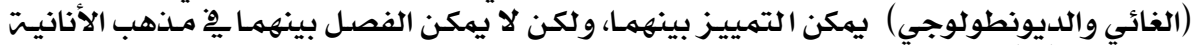

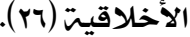

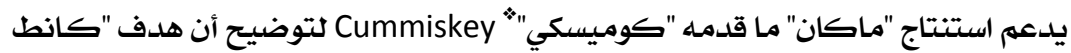

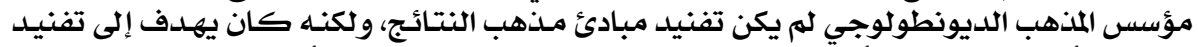

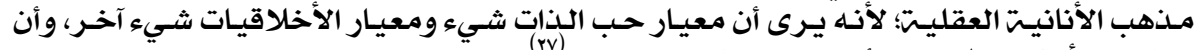

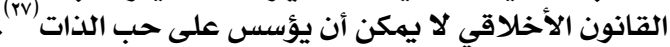

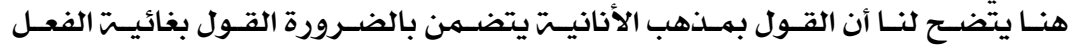
الأخلاقى ولكن من الصعب أن يؤيد الواجب الأخلاقى .

" Auburn " Tibor Richard Machan "

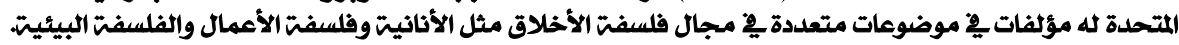
https://mises.org/profile/tibor-r-machan?page=2 16/11/2019

• هو أستاذ الفلسفت بكليت "بايتس" David Cummiskey • https://www.bates.edu/philosophy/files/2010/07/Cummiskey-CV-JUNE2013.pdf 16/11/2019 


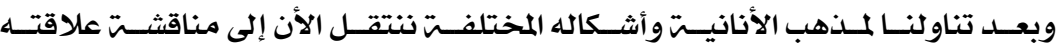

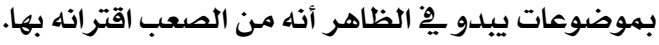

الأنانيت والصد اقنت

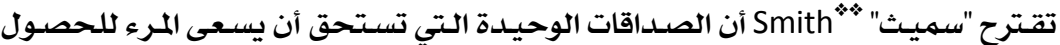

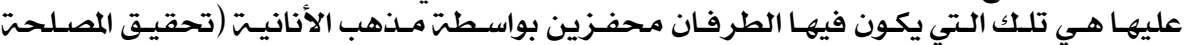

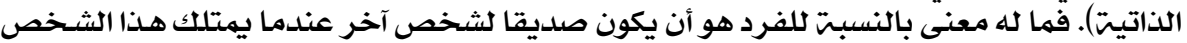

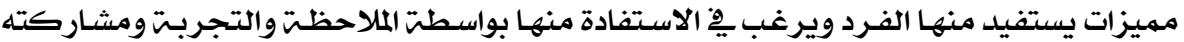

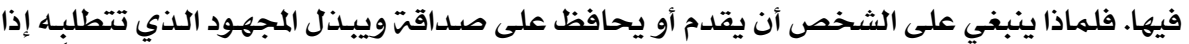

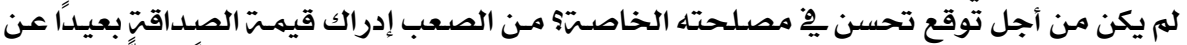

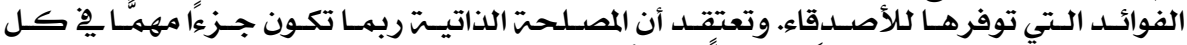

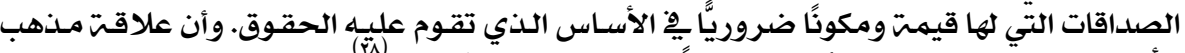

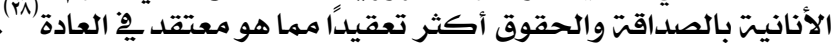

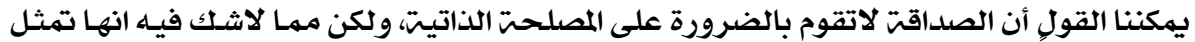

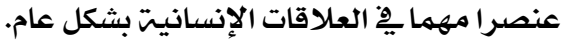

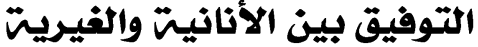

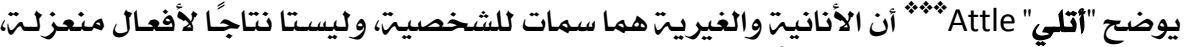

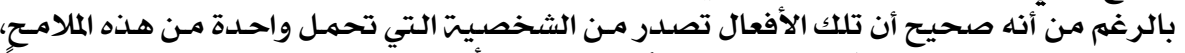

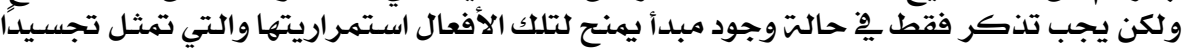

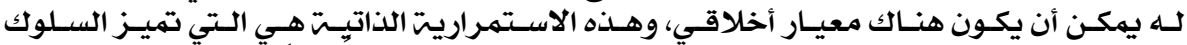

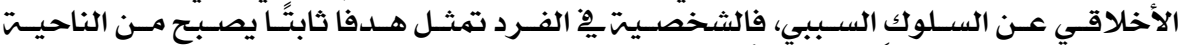

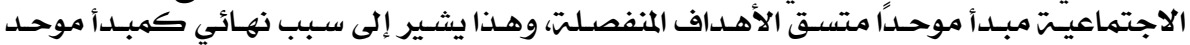

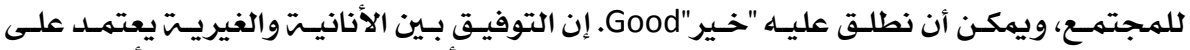

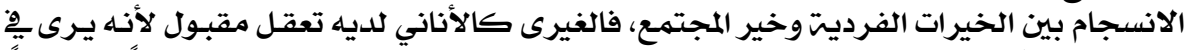

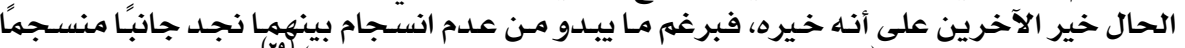

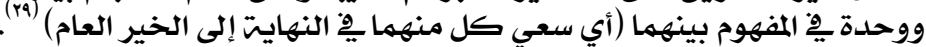

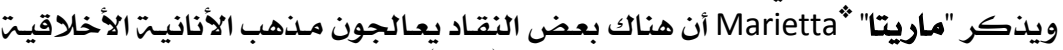

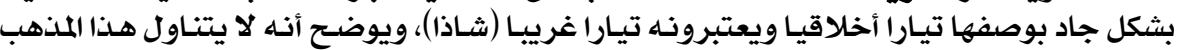

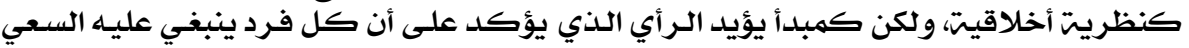

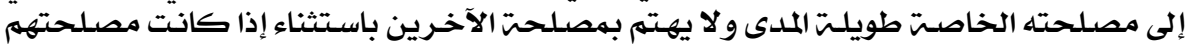

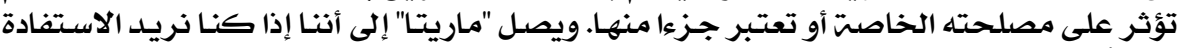

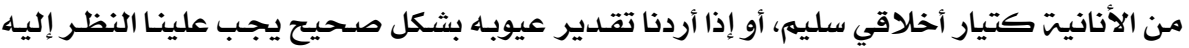

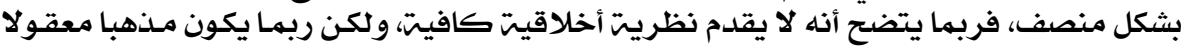

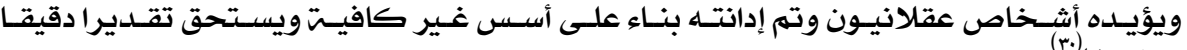

Tara A Smith * * هي أستاذة الفلسفت بجامعت تكساس بالولايات المتحدة الأمريكيت. https://liberalarts.utexas.edu/philosophy/faculty/smithta 16/11/2019

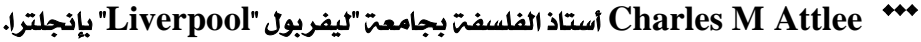

Florida Atlantic هواستاذ الفلسفت بجامعت "فلوريدا أتلانتك "Don E Marietta, Jr • Marietta, Don E. “On Using People ”.Ethics ،vol. 82, no. 3, 1972, pp. 232-

238 JSTOR ،www.jstor.org/stable/2380201 . 17/11/2019 


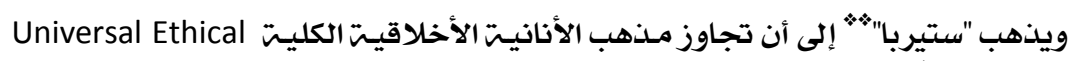

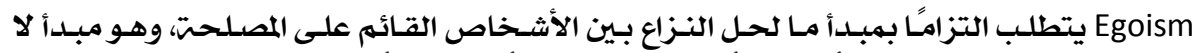

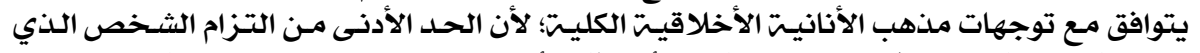

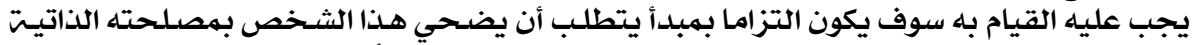

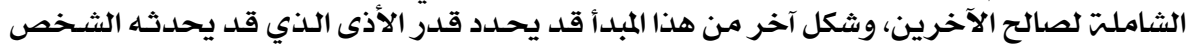

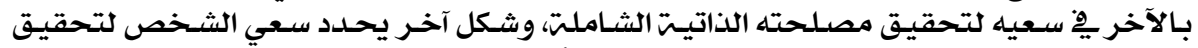

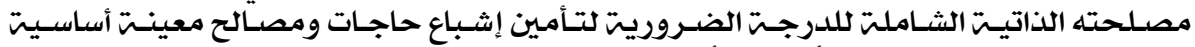

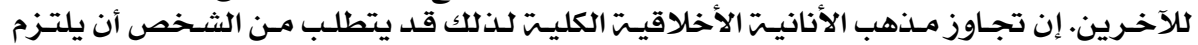

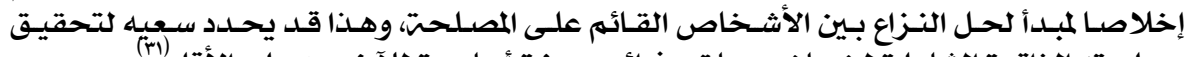

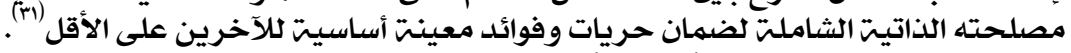

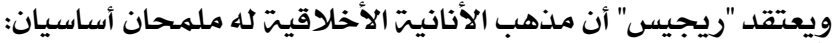

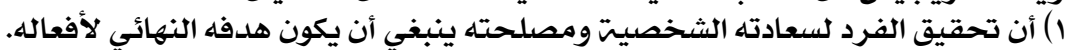

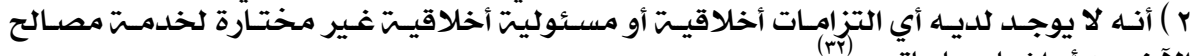

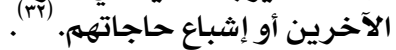

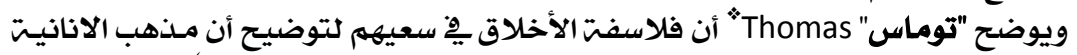

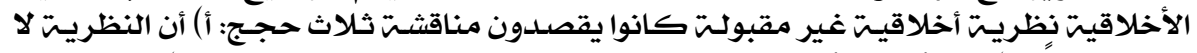

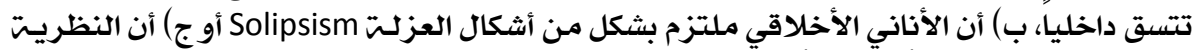

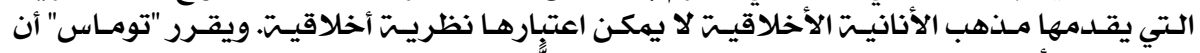

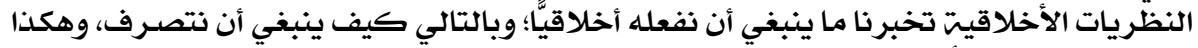

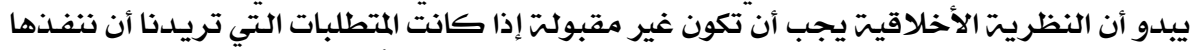

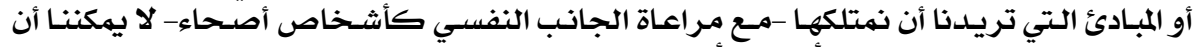

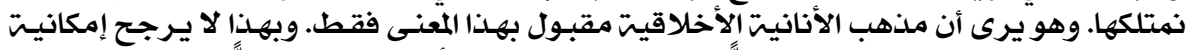

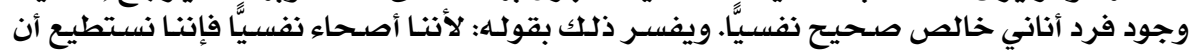

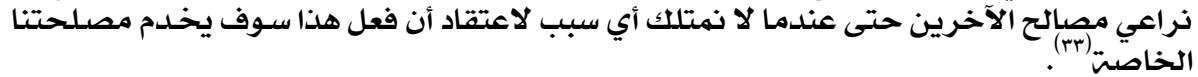

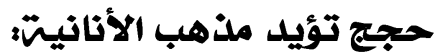 \\ وهناك حجج أخرى يتم تقديمها لصائح مذهب الأنانيت منها:}

حجت الاقتصادي:

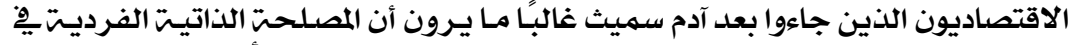

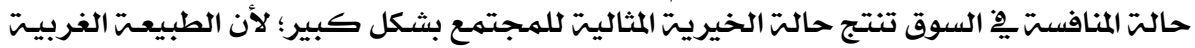

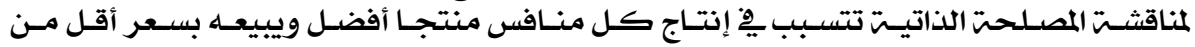
منافسيه. فالمصلحت الذاتيت المستنيرة تقودنا إلى أفضل حالت كلينتة

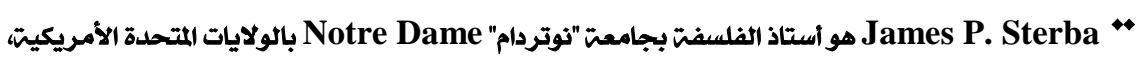
وتولى رئاست العديد من الجمعيات الفلسفيت. https://philosophy.nd.edu/people/faculty/james-sterba/ 22/11/2019

Laurence Thomas • https://www.maxwell.syr.edu/psc/Thomas,_Laurence/23/11/2019

المتحدة الأمريكيت. 


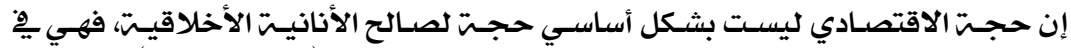

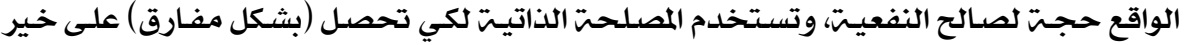

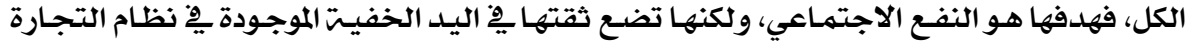

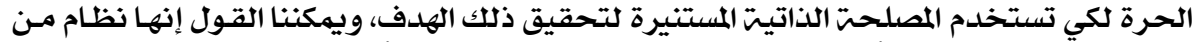

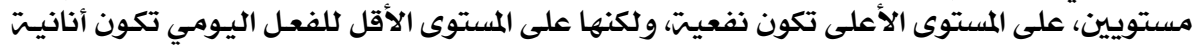

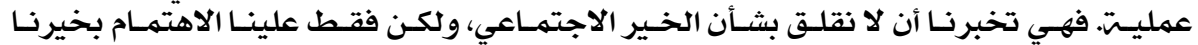

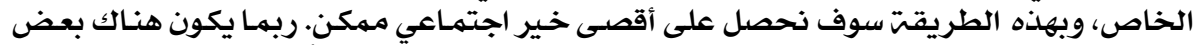

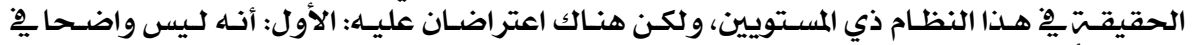

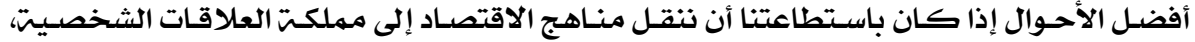

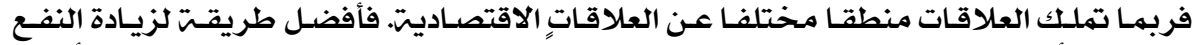

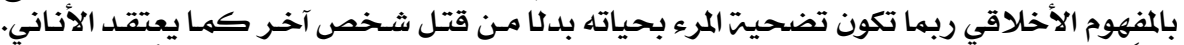

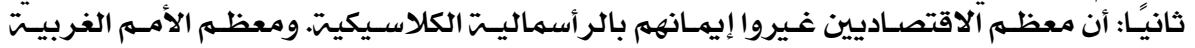

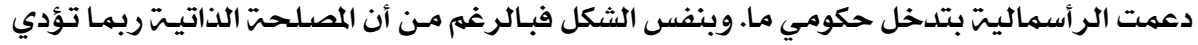

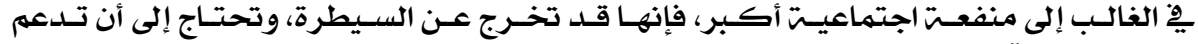

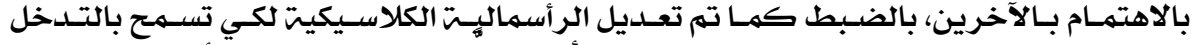

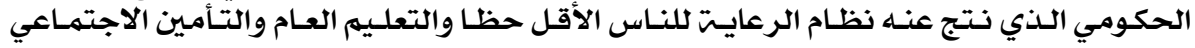
فالنظام الأخلاقي الكايِّ (المعقول) ربما يحتاج إلى إثارة الانتباه إلى احتياجات الآخرين،

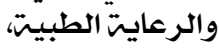

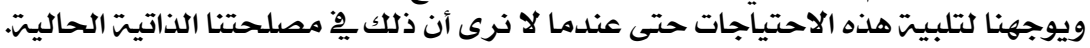

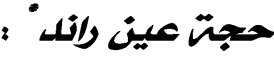

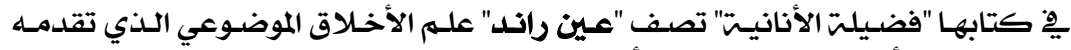

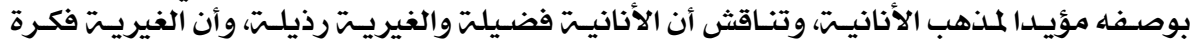

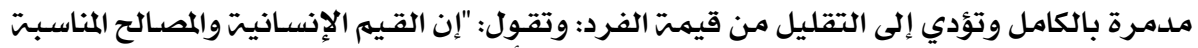

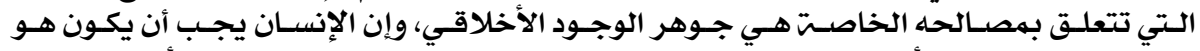

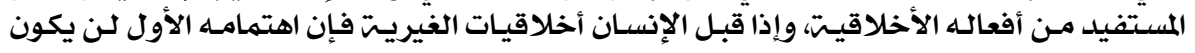

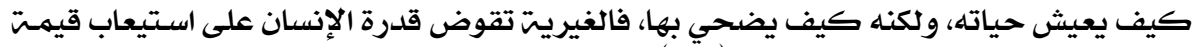

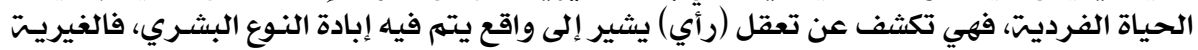

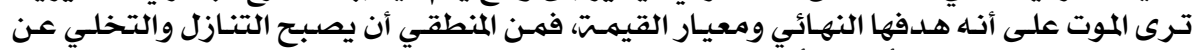

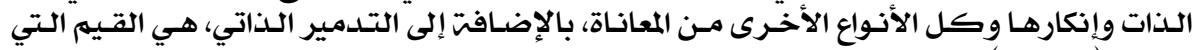
تؤيدها (الغيريتِّ).

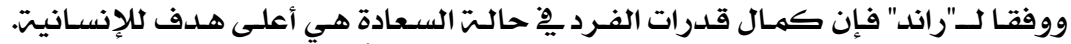

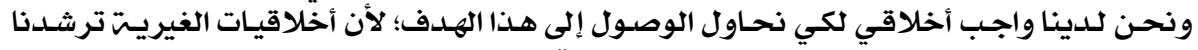

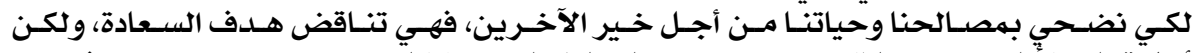

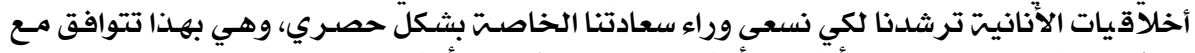

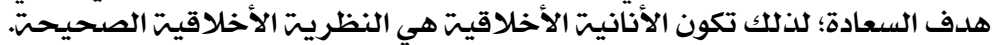

الجني Ayn Rand "

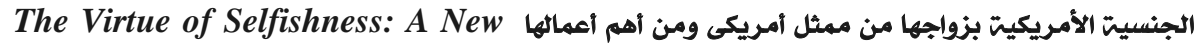
https://plato.stanford.edu/entries/ayn-rand/2/11/2020 Concept of Egoism 


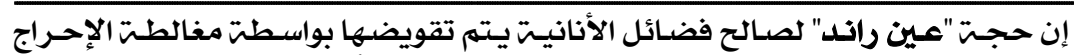

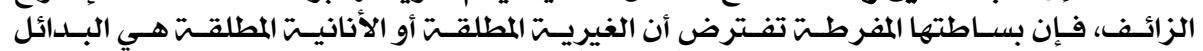

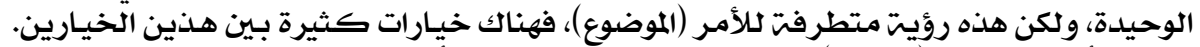

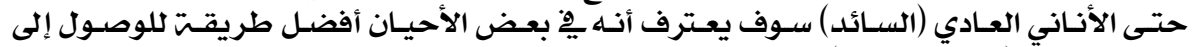

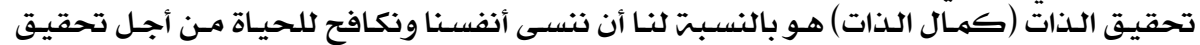

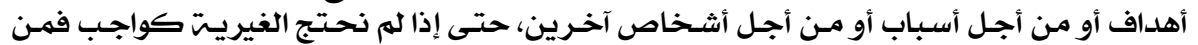

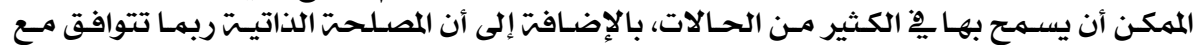

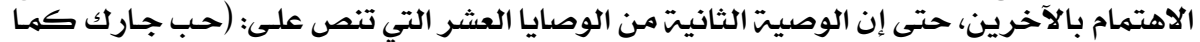

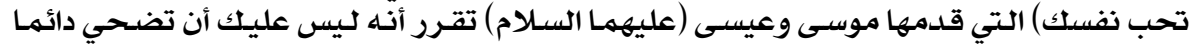

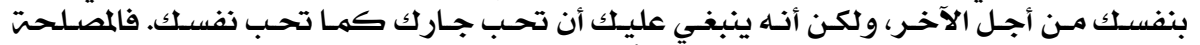

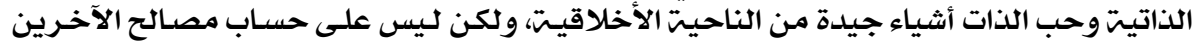

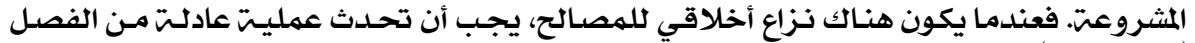

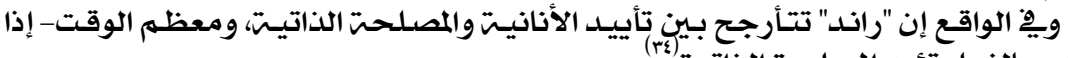
(أو التحكيم) بينها.

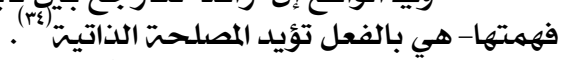

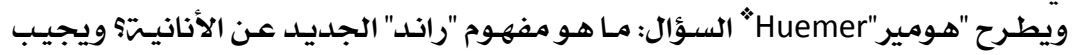

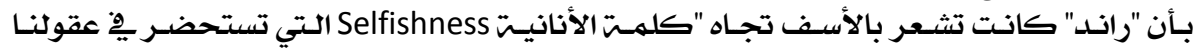

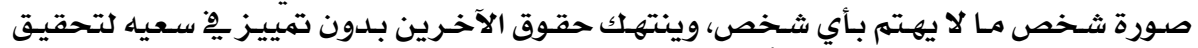

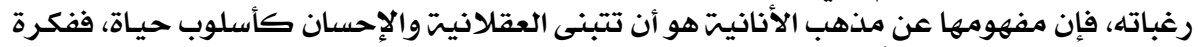

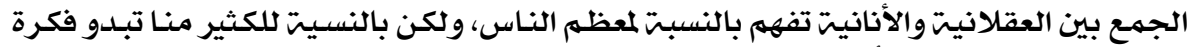

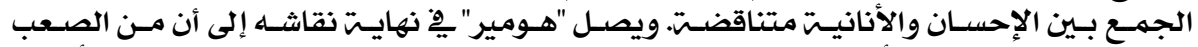

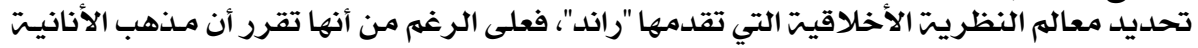

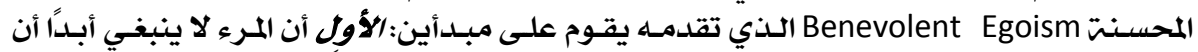

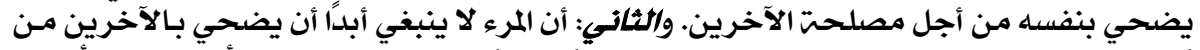

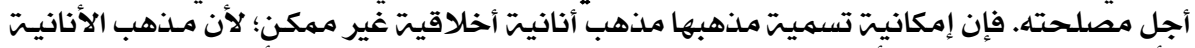

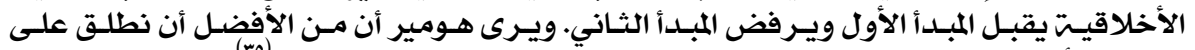

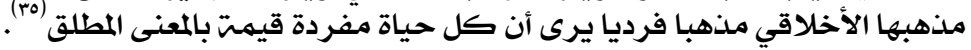

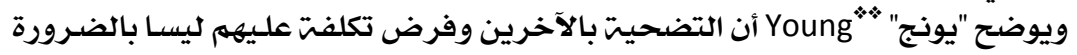

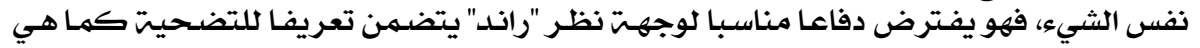

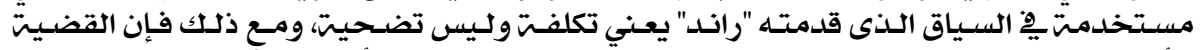

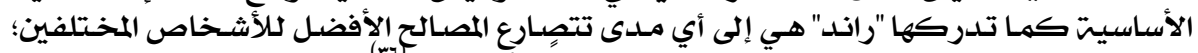

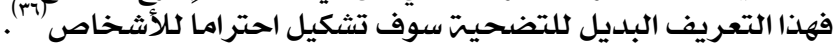

• هو أستاذ الفلسفت بجامعت كلورادو Colorado بالولايات المتحلدة الأمريكيتً. https://colorado.academia.edu/MichaelHuemer 6/2/2020

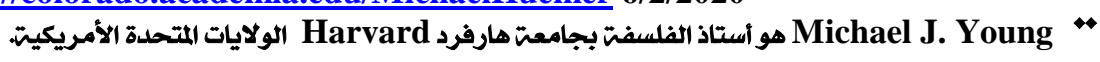
https://www.gatescambridge.org/members-

area/connect/directory/scholar/6406?Sc 6/2/2020 


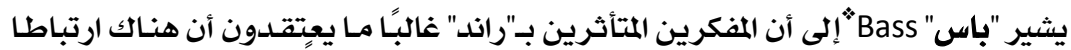

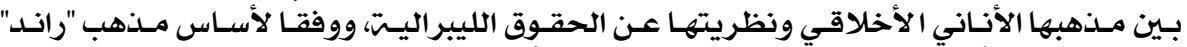

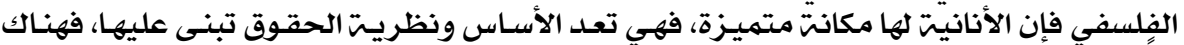

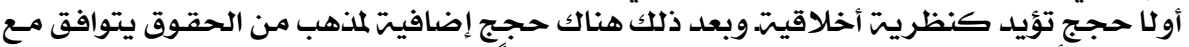

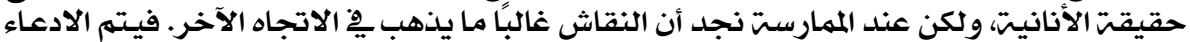

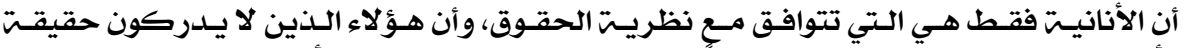

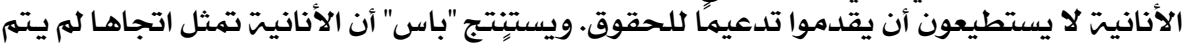

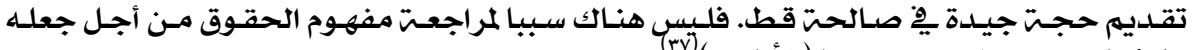

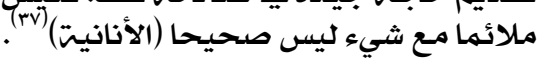

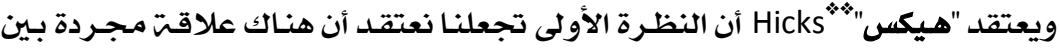

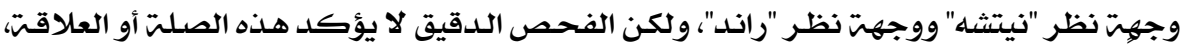

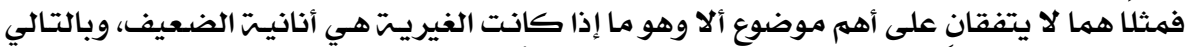

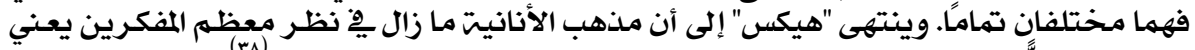

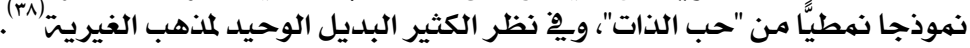

حجن هوبز

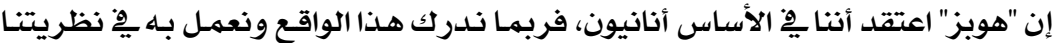

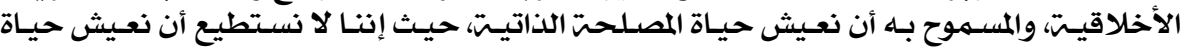

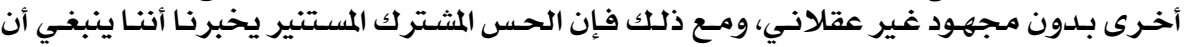

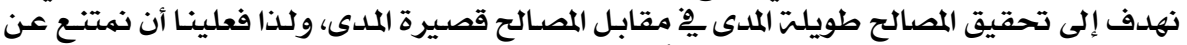

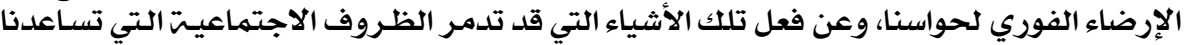

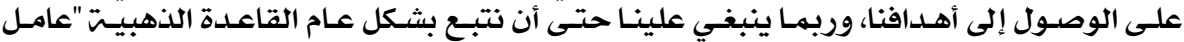

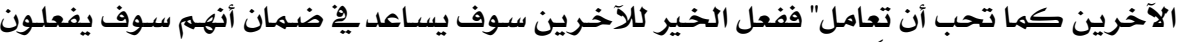

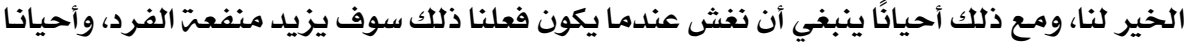

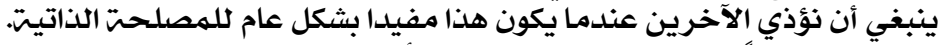

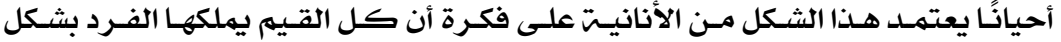

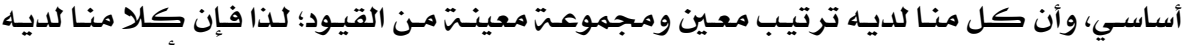

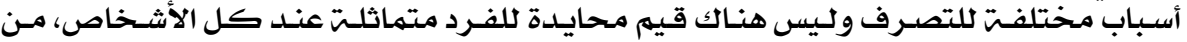

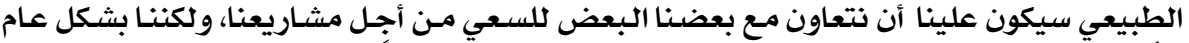

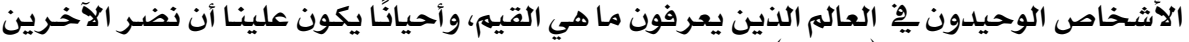
من أجل إدرالك مشاريعنا (أهدافنا).

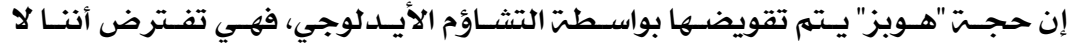

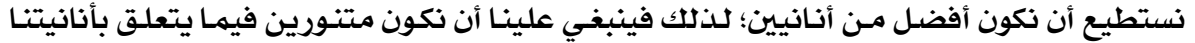

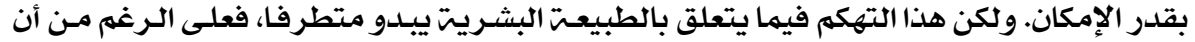

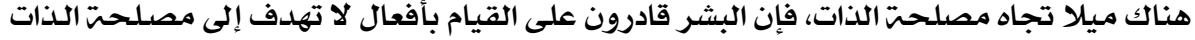

Robert H. Bass * https:/uncp.academia.edu/RobertBass/CurriculumVitae 6/2/2020

الأمريكيت. هو أستاذ الفلسفت بجامعت روكفورد Stephen R. C. Hicks بالولايات المتحدة Rockford

https://atlassociety.org/about-us/staff/21-stephen-hicks 7/2/2020 الأمريكين. 


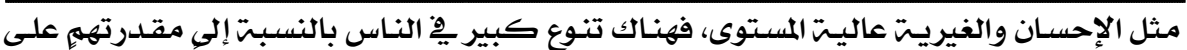

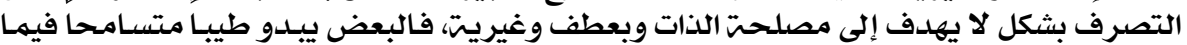

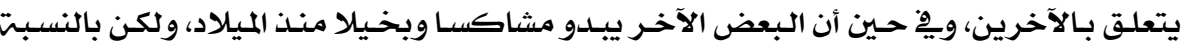

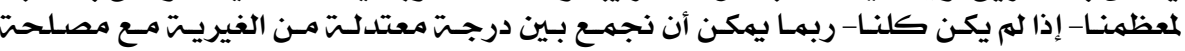
الذذات.

\section{حجج ضد الأنانيت الأخلاقيت}

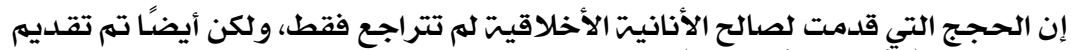

حجج ضد هذا المذهب (الأنانيت الأخلاقيتة) من الهن أهمهما:

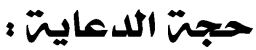

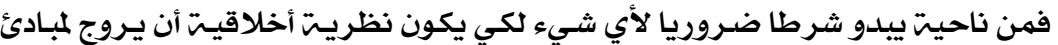

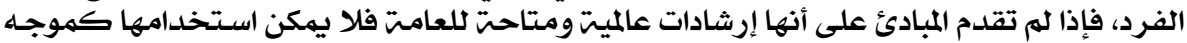

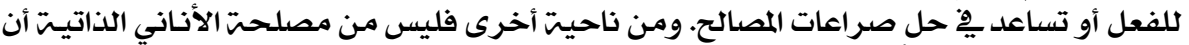

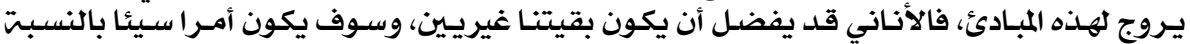

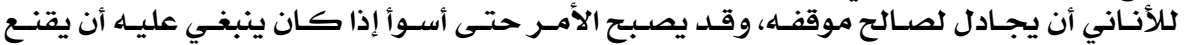

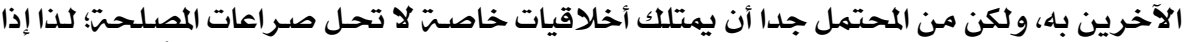

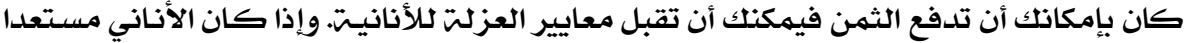

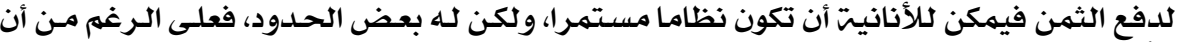

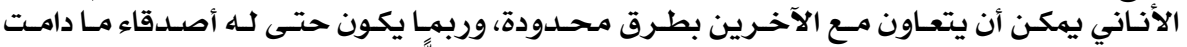

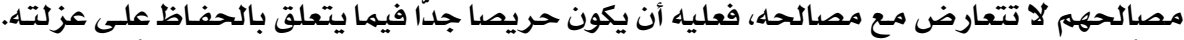

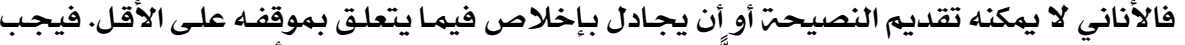

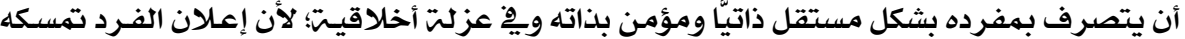

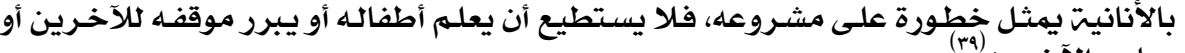

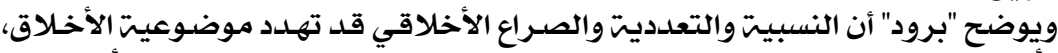

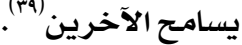

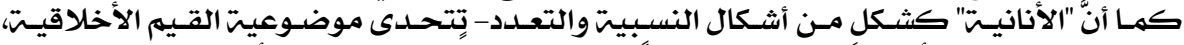

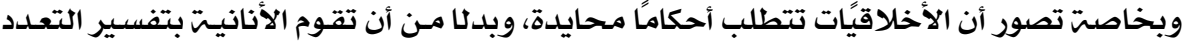

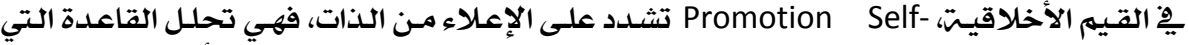

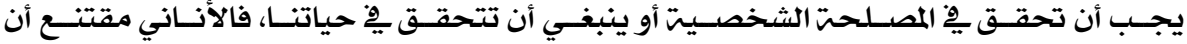

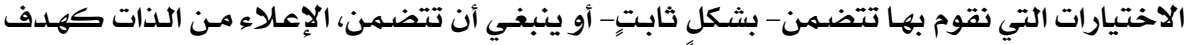

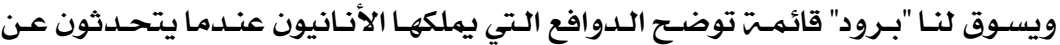

أساسي.

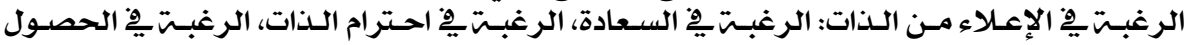

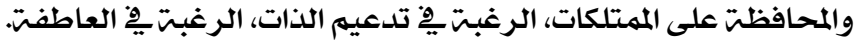
وهناك نوعان من الأنانيتي:

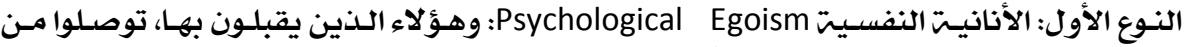

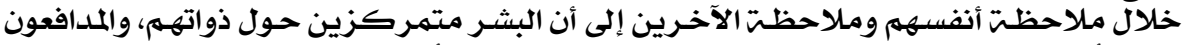

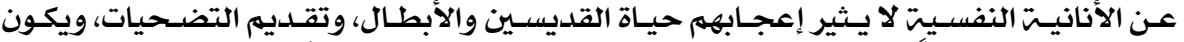

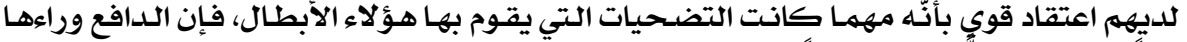

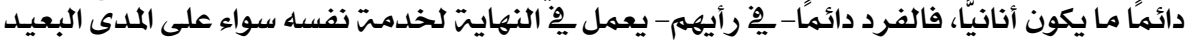

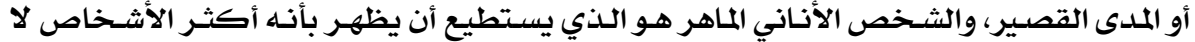

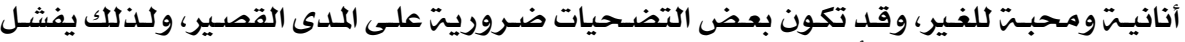
الجدل بالتضحيات ضد ضد الأنانيت. 


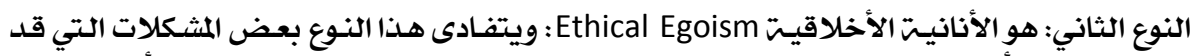

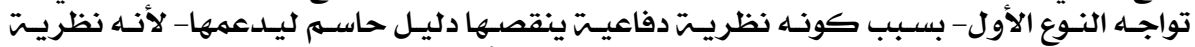

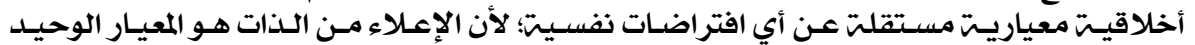

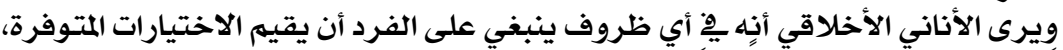
الصحيح للسلوك.

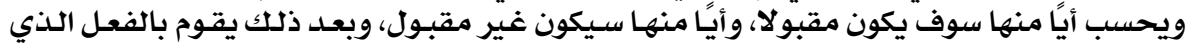

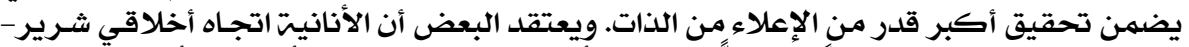

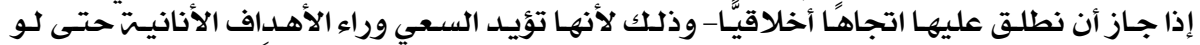

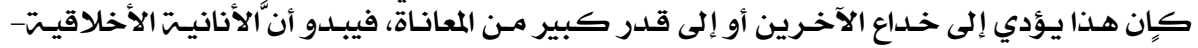

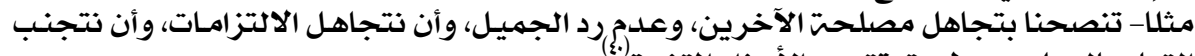

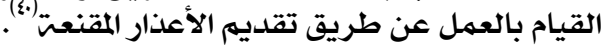

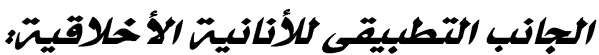

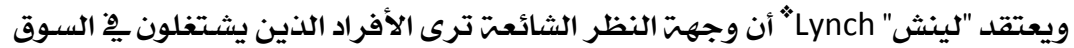

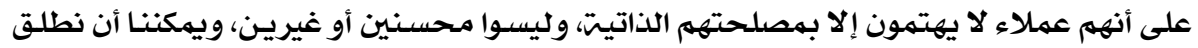

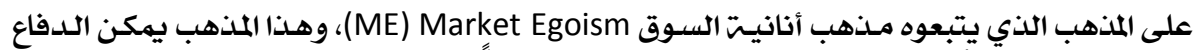

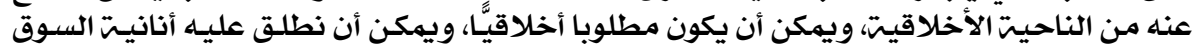

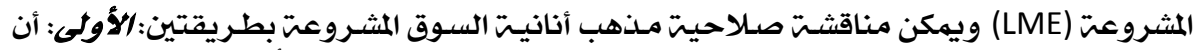

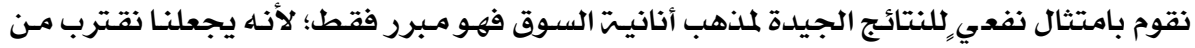

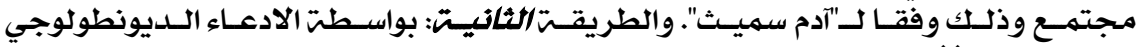

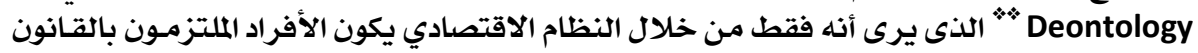

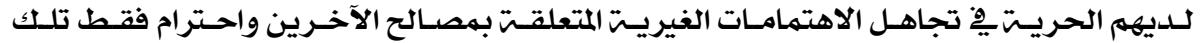

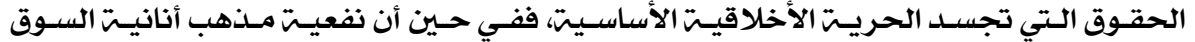

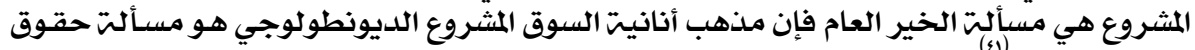
إنسانيت فرديت في (i)

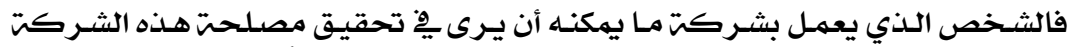

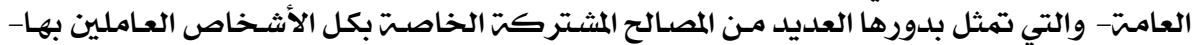
تحقيقا لمصلحته الشخصيتة

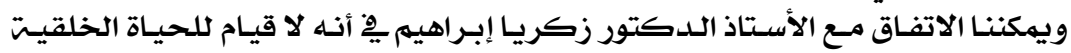

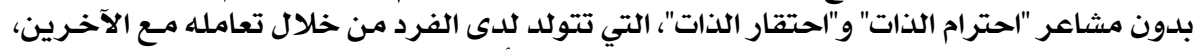

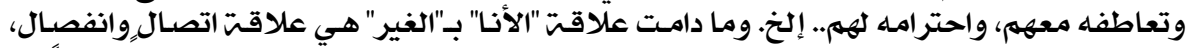

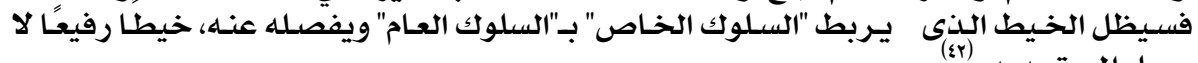

Tony Lynch * https://www.une.edu.au/staff-profiles/hass/alynch 8/2/2020

المذهب أو النظريت الأخلاقيت التي تجعل من الواجب أساسًا للأخلاق. Deontology *

Flew,Antony,Adictionary of philosophy,Updated and RevisedEdition,Macmillan Reference books,1985,p88,89 


\section{الاستنتاج}

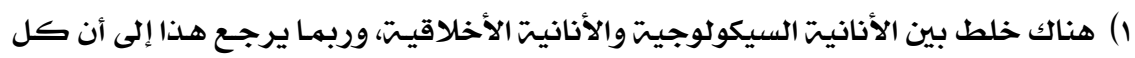

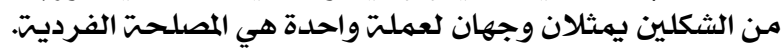

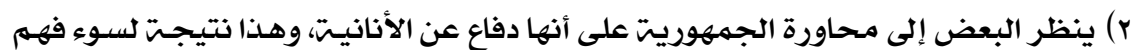

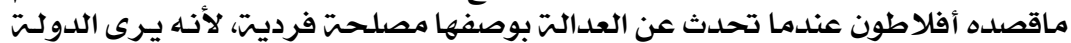
صورة كبيرة للنفس الفرديت.

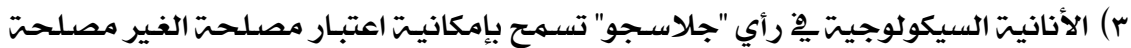
شخصيت.

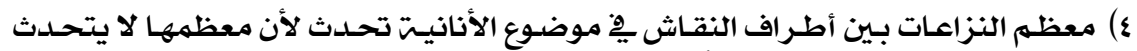

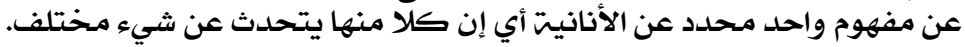

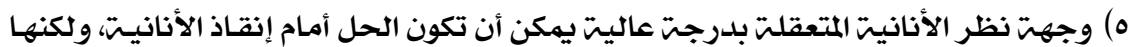

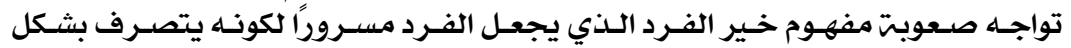

فاضل.

ك) إن التحليل النفسي لا يمدنا دائمًا بالإجابت المناسبتة عن التساؤلات الأخلاقيتض كمها اعتقد صاحب مذهب الأنانيت السيكولوجيتة الإنا

V) بعض المفكرين لا يستبعدون إمكانيت التوفيق بين الأنانيت والغيريت.

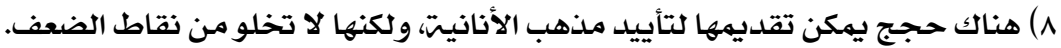
q) الحياة الإنسانيت هي علاقتت مستمرة بين الأنا والغير. 


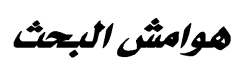

(1) Sisson,Edward O,Egoism,Altruism,Catholism.A Note on Ethical Terminology ,The Journal of Philosophy, Psychology and scientific Methods,Vol.7,No.6,(Mar.17),Journal of philosophy ,1910, p.158:159. \&Spencer,Herbert,The Data of Ethics,William and Norgate,London,1879,p.234.

(2) Edel,Abraham,Two Traditions in The Refutation of Egoism the Journal of philosoph,Vol.34,No.23,(Nov11),Journal of Philosophy,1937,p.617:618

(3) Ibid,p.628.

(4) Lemos,Ramon M.,Psychological Egoism,Philosophy and Phenomenological Research,Vol.20.No.4(Jun),International

Phenomenological Society,1960,p.540\&p.546.

(5) Slote,Michael,AnEmpirical Basis for Psychological Egoism,The Journal of philosophy,Vol.61,No.18,(Oct,1),Journal of philosophy, 1964,P.530:531

(6) Ibid,p.537.

(7) Brandt, Richard,Rationality,Egoism and morality, The Journal of philosophy, Vol.69,No.20,Sixty -Ninth annual meeting of the American philosophical Association Eastern Division (Nov,9),Journal of Philosophy, 1972,p.681

(8) Ibid,p.696.

(9) Barratt,Alfred, The "Suppression" of Egoism,Mind ,Vol2,No.6,(Apr.,) Oxford University Press on behalf of the Mind Association,1877,p.185.

${ }^{(10)}$ Brunton,J.A,Egoism and morality ,The Philosophical Quarterly,Vol.6, No.25(Oct.))Oxford University Press on behalf of the Scots philosophical Association and the university of St,Andrews,1956,P.290

(11) Ibid,p.303.

(12) Nielsen,Kai,Egoism in ethics,Philosophy and Phenomenology Research, Vol.19,No.4(Jun),Published by :international phenomenological Society,1959,p.502

(13) Ibid,p.510.

(14) Gert,Bernard,Hobbes,Machanism,and Egoism,The philosophical Quarterly ,Vol.15,No.16(Oct),Oxford university Press on behalf of the Scots philosophical Association and the University of St.Andrews, 1965,p.341,p.349

${ }^{(15)}$ Versenyi,Laszlo,ls Ethical Egoism really inconsistent? Ethics,Vol.80, No.3(Arp.),),the university of Chicago press,1970,p.241 
${ }^{(16)}$ Glasgow,W.d,Ethical Egoism again,Ethics,Vol.82,No.(Oct,)The University of Chicago press,1971,p.66,p.71

(iv) \& Kraut,Richard,Egoism,Love and Political office in Plato,The philosophical Review,Vol.82,No.3(Jul.),Duk University press on behalf of Philosophical Review,1973,p.330,p.343.

${ }^{(18)}$ Broad C.D,"Egoism as A theory of Human Motives" in Ethics and the history of philosophy,London:Routledg\&Kegan paul,1952,p.220.

${ }^{(19)}$ Glasgow,W.D,Broad on Psychological Egoism,Ethics,Vol.88,No.4(Jul.),The University of Chicago press,1978,p.361:362,p.368

${ }^{(20)}$ Facion,peter A,Donald Scherer,and Thonas Atting,Values and Socitey,Englewood Cliffs,NJ.:Prentice-Hall,Inc,1978,311

${ }^{(21)}$ Regis,Edward,What is Ethical Egoism?,Ethics,Vol.91.,No.1,The University of Chicago Press,1980.p.50:51,p.62.

(22) Lemos,Noah M,High-Minded Egoism and the Problem of Priggishness, Mind,New Series,Vol.93,No.379(Oct),Oxford University Press on behalf of The Mind Association,1984,P.542:543,P.558

${ }^{(23)}$ Margolis,Joseph,Egism and the Confirmation of metamoral Theories, American Philosophical Quarterly,Vol.7,No.3(Jul.),University of Illionois press on behalf of The North American philosophical Publications,1970,p.260.

${ }^{(24)}$ Glasgow,W.D,Psychological Egoism,American philosophical Quarterly,Vol.13,No.1(Jan) ,University of llionois press on behalf of The North American philosophical Publications,1976,p.75.

(25) Brown,Norman J,Psychological Egoism Revisited ,philosophy,Vol.54, No.209(Jul),Cambridge University press on behalf of Royal Institute of Philosophy,1979,P.293.

${ }^{(27)}$ Cummiskey,David,Consequentialism,Egoism,and The Moral Law, Philosophical Studies:An International Journal for Philosophy in the Analytic Tradition,Vol.57,No.2(Oct.),),Springer,1989,p.132

(28) Smith,Tara,Right ,Friends,and Egoism, The Journal of Philosophy,Vol.90,No.3 (Mar),Journal of philosophy,1993,p.148.

(29) Attlee,Charkes M,Egoism, The Monist,Vol.38,No.4,Oct,Oxford University Press,1928,P.567:568. 
(30) Marietta.Jr,Don E, The Alleged oddness of ethical Egoism,Journal of Thought,Vol.12.No.4(Nov),Caddo Gap Press,1977,p.280

(31) Sterba,James P.,Ethical Egoism and Beyoned,Canadian Journal of philosophy,Vol.9,No.1(Mar.),Canadian Journal of philosophy,1979,P.108.

${ }^{(32)}$ Regis,Edward,Ethical Egoism and Moral Responsibility,American Quarterly,Vol.16,No.1,(Jan), University of Illionois press on behalf of The North American philosophical Publications,1979,p.45,p.47,p.51.

(33) Thomas,Laurence,Ethical Egoism and psychological dispositions, American PhilosophicalQuarterly,Vol.17,No.1(Jan),University of Illionois press on behalf of The North American philosophical Publications,1980,p.37,p.78

(34)Pojman,Louis P, Philosophy The pursuit of wisdom,third edition,Wadsworth,USA,2001,p.271. \&Rand,Ayn,The Virtues of Selfishness,Signet Books,New York,1964,p.27-34.

(35) Humer,Michael, Is benevolent egoism coherent?,The Journal of Ayn Rand studies,Vol.3,No.3(Spring),Penn Stste University Press, 2002,p.259,285

(36) Young,Michael,Reply to Michael Huemer's "Is benevolent egoism coherent? ",(spring) on Egoism and predatory behavior,The Journal of Ayn Rand Studies Vol.5,No.2 (Spring) ,Penn State University Press, 2004,p.456.

(37) Bass,Robert H.,Egoism Versus Rights,The Journal of Ayn Rand studies,Vol.7,No.2 (Spring),Penn state University Press,2006,p.329,345

${ }^{(38)}$ Hicks, Stephen R. C,Egoism in Nietzsche and Rand,The Journal of Ayn Rand studies, Vol.10,No.2,Symposium on Friedrich Nietzsche\& Ayn Rand(Spring), Penn state University Press,2009,p.287

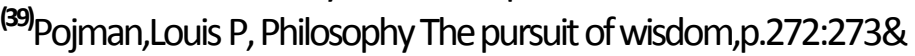

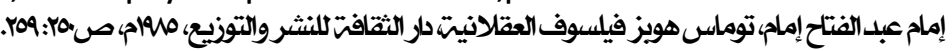

(40) Broad ,C.D,"Ethics and the history of philosophy "Routledge\& Keganpaul London ,1952“, pp 218-230.

(41) Lynch,Tony, Legitimating market Egoism: The Availability problem, Journal of Business Ethics,Vol.84,No.1(Jan),Springer,2009,p.8.

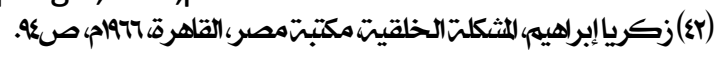




\section{مراجع البحث}

1- Attlee,Charkes M,Egoism,The Monist,Vol.38,No.4,Oct,Oxford University Press,1928.

2- Barratt,Alfred, The "Suppression" of Egoism,Mind,Vol2,No.6,(Apr.,) Oxford University Press on behalf of the Mind Association,1877

3- Bass,Robert H.,Egoism Versus Rights, The Journal of Ayn Rand studies,Vol.7,No.2 (Spring),Penn state University Press,2006.

4- Brandt, Richard,Rationality,Egoism and morality, The Journal of philosophy, Vol.69,No.20,Sixty -Ninth annual meeting of the American philosophical Association Eastern Division (Nov,9),Journal of Philosophy, 1972

5- Broad ,C.D,"Ethics and the history of philosophy " Routledge\& Keganpaul London, 1952.

6- Broad C.D," Egoism as A theory of Human Motives" in Ethics and the history of philosophy,London:Routledg\&Kegan paul,1952.

7- Brown,Norman J,Psychological Egoism Revisited ,philosophy,Vol.54, No.209(Jul),Cambridge University press on behalf of Royal Institute of Philosophy,1979.

8- Brunton,J.A,Egoism and morality ,The Philosophical Quarterly,Vol.6, No.25(Oct.))Oxford University Press on behalf of the Scots philosophical Association and the university of St,Andrews,1956.

9- Cummiskey,David,Consequentialism,Egoism, and The Moral Law,Philosophical Studies:An International Journal for Philosophy in the Analytic Tradition,Vol.57,No.2(Oct.),),Springer,1989.

10- Edel,Abraham, Two Traditions in The Refutation of Egoism, the Journal of philosoph,Vol.34,No.23,(Nov11),Journal of Philosophy,1937.

11- Facion,peter A,Donald Scherer,and Thonas Atting,Values and Socitey,Englewood Cliffs,N.J.:Prentice-Hall,Inc,1978.

12- Gert,Bernard,Hobbes,Machanism, and Egoism,The philosophical Quarterly ,Vol.15,No.16(Oct),Oxford university Press on behalf of the Scots philosophical Association and the University of St.Andrews, 1965. 
13- Glasgow,W.d,Ethical Egoism again,Ethics,Vol.82,No.(Oct,)The University of Chicago press,1971.

14- Glasgow,W.D,Psychological Egoism,American philosophical Quarterly,Vol.13,No.1(Jan) ,University of Illionois press on behalf of The North American philosophical Publications,1976.

15- Glasgow,W.D,Broad on Psychological Egoism,Ethics,Vol.88 No.4(Jul.),The University of Chicago press,1978.

16- Hicks, Stephen R. C,Egoism in Nietzsche and Rand,The Journal of Ayn Rand studies, Vol.10,No.2,Symposium on Friedrich Nietzsche\& Ayn Rand(Spring), Penn state University Press,2009.

17- Humer,Michael, Is benevolent egoism coherent?,The Journal of Ayn Rand studies,Vol.3,No.3(Spring),Penn Stste University Press,2002.

18- Kraut,Richard,Egoism,Love and Political office in Plato,The philosophical Review,Vol.82,No.3(Jul.),Duk University press on behalf of Philosophical Review,1973

19- Lemos,Noah M,High-Minded Egoism and the Problem of Priggishness, Mind,New Series,Vol.93,No.379(Oct.),Oxford University Press on behalf of The Mind Association,1984

20- Lemos,Ramon M.,Psychological Egoism,Philosophy and Phenomenological Research,Vol.20.No.4(Jun),International Phenomenological Society,1960.

21- Lynch,Tony, Legitimating market Egoism: The Availability problem, Journal of Business Ethics,Vol.84,No.1(Jan),Springer,2009.

22- Margolis,Joseph,Egism and the Confirmation of metamoral Theories, American Philosophical Quarterly,Vol.7,No.3(Jul.),University of Illionois press on behalf of The North American philosophical Publications,1970.

23- Marietta.Jr,Don E, The Alleged oddness of ethical Egoism,Journal of Thought,Vol.12.No.4(Nov),Caddo Gap Press,1977.

24- Nielsen,Kai,Egoism in ethics,Philosophy and Phenomenology Research,Vol.19,No.4(Jun),Published by :international phenomenological Society,1959. 
25- Pojman,Louis P, Philosophy The pursuit of wisdom,third edition,Wadsworth,USA,2001.

26- Rand,Ayn,The Virtues of Selfishness,Signet Books,New York,1964

27- Regis,Edward,Ethical Egoism and Moral Responsibility,American Quarterly,Vol.16,No.1,(Jan), University of Illionois press on behalf of The North American philosophical Publications,1979.

28- Regis,Edward,What is Ethical Egoism?,Ethics,Vol.91.,No.1,The University of Chicago Press,1980.

29- Sisson,Edward O,Egoism,Altruism,Catholism.A Note on Ethical Terminology ,The Journal of Philosophy, Psychology and scientific Methods,Vol.7,No.6,(Mar.17),Journal of philosophy,1910.

30- Smith,Tara,Right, Friends, and Egoism,The Journal of Philosophy, Vol.90,No.3 (Mar),Journal of philosophy ,1993.

31- Spencer,Herbert,The Data of Ethics,William and Norgate,London,1879.

32- Sterba,James P.,Ethical Egoism and Beyoned,Canadian Journal of philosophy,Vol.9,No.1(Mar.),Canadian Journal of philosophy,1979.

33- Thomas,Laurence,Ethical Egoism and psychological dispositions, American PhilosophicalQuarterly,Vol.17,No.1(Jan),University of Illionois press on behalf of The North American philosophical Publications,1980.

34- Versenyi,Laszlo,Is Ethical Egoism really inconsistent?,Ethics,Vol.80, No.3(Arp.),)the university of Chicago press, 1970.

35- Young,Michael,Reply to Michael Huemer's "Is benevolent egoism coherent? ",(spring) on Egoism and predatory behavior,The Journal of Ayn Rand Studies Vol.5,No.2 (Spring) ,Penn State University Press,2004,

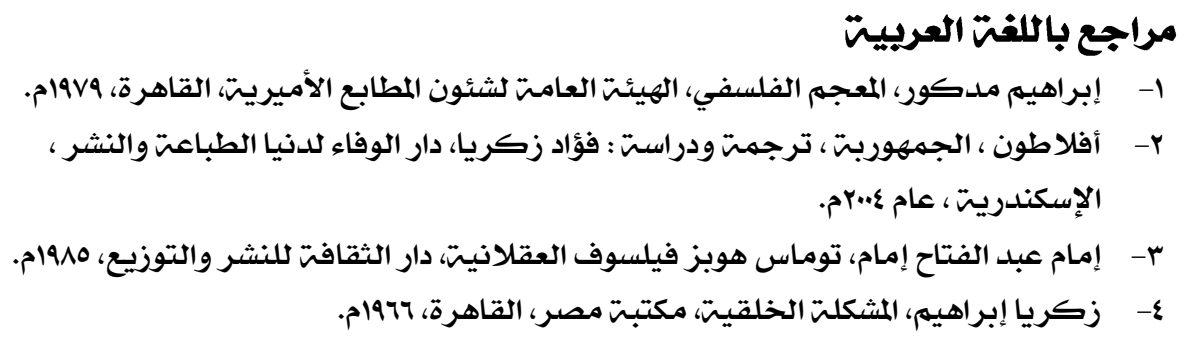


1-https://plato.stanford.edu/entries/egoism/

$$
\text { مواقع على شبكت المعلومات الدوليتي: }
$$

2-https://www.umt.edu/president/people/pastpresidents/siss on.php

3-https://www.oxfordreference.com/view/10.1093/oi/authorit y.20110915125649353

http://web.gc.cuny.edu/Philosophy/edel.html

4-http://www.phil.arts.cuhk.edu.hk/web/tcivp/michael-slote

5-https://www.ukwhoswho.com/search?q=Austin+DuncanJones\&searchBtn=Search\&isQuickSearch=true

6-https://www.cambridge.org/core/services/aop-cambridgecore/content/view/S0953820800006300

7-https://oxfordindex.oup.com/oi/viewoverview/10.1093\$002f oi\$002fauthority.20110803095448519

8-https://phil.ucalgary.ca/profiles/kai-nielsen

9-http://www.chapelhillnews.com/2012/01/01/69116/bernard -gert.html

10- https://www.nytimes.com/1988/02/26/obituaries/laszlo-gversenyi-philosophy-professor-59.htm

11- https://plato.stanford.edu/entries/broad/

12- http://independent.academia.edu/PeterFacione/Curriculum Vitae

13- https://www.bgsu.edu/arts-andsciences/philosophy/faculty-and-staff/dschere.html

14- https://www.britannica.com/biography/A-C-Ewing

15- https://liberalarts.temple.edu/academics/faculty/margolisjoseph?action

16- https://mises.org/profile/tibor-r-machan?page=2

17- https://www.bates.edu/philosophy/files/2010/07/Cummisk ey-CV-JUNE-2013.pdf

18- https://liberalarts.utexas.edu/philosophy/faculty/smithta 
19- https://philosophy.nd.edu/people/faculty/iames-sterba/

20- https://www.maxwell.syr.edu/psc/Thomas, Laurence/

21- https://colorado.academia.edu/MichaelHuemer

22- https://www.gatescambridge.org/membersarea/connect/directory/scholar/6406?Sc

23- https://uncp.academia.edu/RobertBass/CurriculumVitae

24- https://atlassociety.org/about-us/staff/21-stephen-hicks

25- https://www.une.edu.au/staff-profiles/hass/alync 